\title{
Pre-main sequence candidates in the very young open cluster NGC 6611
}

\author{
D. de Winter ${ }^{1,3}$, C. Koulis ${ }^{1}$, P.S. Thé ${ }^{1}$, M.E. van den Ancker ${ }^{1}$, M.R. Pérez ${ }^{2}$, and E.A. Bibo ${ }^{1}$ \\ 1 Astronomical Institute "Anton Pannekoek", University of Amsterdam, Kruislaan 403, 1098 SJ Amsterdam, The Netherlands \\ 2 Applied Research Corp., Suite 1120, 8201 Corporate Dr., Landover, MD 20785, U.S.A. \\ 3 Dpto. Física Teórica, C-XI, Facultad de Ciencias, Universidad Autónoma de Madrid, Cantoblanco, E-28049 Madrid, Spain
}

Received February 19; accepted May 22, 1996

\begin{abstract}
For the search of Herbig Ae/Be objects in the extremely young open cluster NGC 6611 we have selected a sample of 52 pre-main sequence candidates, discovered by Walker (1961), Sagar \& Joshi (1979), Chini \& Wargau (1990) and Thé et al. (1990). We continue the approach of the last paper by studying each star individually with new and unpublished Walraven $W U L B V$, Johnson/Cousins $U B V(R I)_{\mathrm{C}}$ and Johnson $J H K L M$ photometric data as well as low resolution spectroscopy.

Each object is shown to have its own extinction law, which is investigated using their spectral energy distribution (SED). There does not seem to be a clear relationship between the location of a star and the extinction law. This means that the extinction is generated locally and its correction must be taken individually. For each object accurate astrophysical parameters are then derived. Plotting the objects in an HR-diagram, together with the values for the $E(B-V)$, the probability of membership value $P$ and the extinction characteristics, helps to discriminate between cluster members and non-cluster members.

Most foreground stars are of late spectral type and are labeled as Group III objects. Group I, to which most members of this cluster belong, contains objects of early spectral type. Part of them seem to be in their postZAMS phase and the other part in their pre-ZAMS stage. By comparing the evolutionary tracks of Palla \& Stahler (1993) for pre-MS objects and of Maeder \& Meyenet (1988) for post-MS stars we have concluded that the cluster contains objects of a few $0.1 \mathrm{Myr}$ as well as objects of about 6 Myr. As most of the Group I objects do not show well-known Herbig Ae/Be characteristics, the time
\end{abstract}

Send offprint requests to: D. de Winter (dolf@astro1.ft.uam.es) * Based partly on observations obtained at the European Southern Observatory, La Silla, Chile. Tables 1-4 and 6 are only available in electronic form at the CDS via anonymous ftp to cdsarc.u-strasbg.fr (130.79.128.5) or via http://cdsweb.ustrasbg.fr/ Abstract.html. Figures 1 and 3 and Sect. 5 are only available in electronic form at the Editions de Physique http://www.ed-phys.fr scale of clearing the disk material must be typically less than about $0.1 \mathrm{Myr}$ for the more massive objects. Objects that show an IR-excess are found among the less luminous ones. They could still be in their pre-ZAMS phase, having an age of about 1 Myr. Such an age is appropriate for the Group II objects, which are of intermediate spectral type. As they are located close to the stellar birthline they should have been formed recently. It seems that an efficient clearing mechanism must have taken place, because not many of these objects are embedded and show only some IR-anomalies. This could be the reason that we have found no more than four typical Herbig Ae/Be candidates.

We support the hypothesis of Hillenbrand et al. (1993) that there is an age spread in NGC 6611. The stars with the highest $A_{V}$ values are located in the centre of the cluster, somewhat to the northwest. This coincides with the location of many embedded sources more towards the northwest, a region in which star-formation is probably still taking place. The age of the most evolved objects is about 6 Myr.

It would be interesting to study these regions and the Group II objects since investigating the youngest objects which are still partly embedded may help us to understand the first phases of star formation and the rapid cleaning of the circumstellar material. Young "naked" stars can also be formed by evaporating gaseous globules (EGGs) as was recently discovered in dark regions of this cluster (Hester $\&$ Scowen 1995). The lack of angular momentum of such objects could explain the paucity of HAeBe candidates.

Key words: circumstellar matter - stars: emission-line — stars: evolution — stars: formation — stars: pre-main sequence - open clusters and associations: NGC 6611 


\section{Introduction}

The open cluster NGC 6611 (see Fig. 8) is located in the constellation Serpens, embedded in the Sagittarius spiral arm. It is part of the North-West of M 16 ("The Eagle Nebula"), an extensive bright emission nebulosity with a large gap in the middle of its northern side. It has a system of sharply defined dark markings (dense dust clouds) of which the most conspicuous is located at the southern side, extending inward into the centre. The nebulosity along the northern rim of this great dark marking is brighter than in the other regions.

Besides the "classical" North-West population of M 16, which we refer to as the "centre", see Figs. 7a-b, NGC 6611 is nowadays assumed to range all over the M 16 area. As M 16 is a star forming region, dark clouds obscure the stars lying behind them. When star formation is taking place the young stellar objects (YSOs) are normally first seen at the edges of dark clouds. Many embedded objects were identified in the near-IR by Hillenbrand et al. (1993) in or near the dark central regions of M 16. In a later phase of the young star a bright reflection nebula can appear. In the case of a nebulous region, such as M 16, the emitted light of these newly born stars will heat up and ionize their surrounding circumstellar (CS) material which can be seen as bright nebulae. Because the cluster NGC 6611 is indeed associated with the star forming region $\mathrm{M}$ 16, its stellar members must be very young.

It is also possible that these young objects are the origin of a strong radiation field of which the UV part will evaporate parts of the dark nebular regions more efficiently than other parts, causing the existence of the socalled Evaporating Gaseous Globules (EGGs, Hester \& Scowen 1995). The impact of the discovery of such EGGs on the star formation (SF) process in NGC 6611 will be discussed in a subsequent paper (Pérez et al. 1997, in preparation).

For the study of pre-main sequence (PMS) stars, very young open clusters are most suitable, because: (a) all cluster members are located at about the same distance. This distance can be well determined employing the spectrophotometric method to early type members, in which the influence of individual anomalous extinction, if any, is taken into account (see Thé et al. 1990); (b) the foreground interstellar extinction can be determined using the early type members (which are undoubtedly true cluster members). This is necessary since the extinction in the direction of PMS objects is due to foreground as well as circumstellar material and the latter often obeys a different extinction law than the former; (c) all cluster members are young, therefore confusion with evolved objects is not to be expected.

One of the first complete studies on NGC 6611 was done by Walker (1961). He constructed the HertzsprungRussell Diagram (HRD) of NGC 6611 and found that it consists of a main sequence (MS) extending from $\mathrm{O} 5$ to about B5, beyond which stars are lying above the MS. The photometric observations of Walker show that many of the stars in NGC 6611 are reddened by an extremely variable amount, which is roughly correlated with their position in the cluster. Northward the extinction is larger and more irregular, southward the colour excess is less.

After Walker's study many investigations followed, e.g.: Johnson \& Borgman (1963), Turner (1974), Sagar \& Joshi (1979), Neckel \& Chini (1981), Chini \& Krugel (1983), Thé et al. (1990). The major conclusion in these studies is that the extinction law in the star forming regions of NGC 6611 is anomalous. The importance of the anomalous extinction (caused by intracluster matter or by circumstellar dust) was recognized because of its influence on the determination of the distance to the cluster, and its effect on the astrophysical parameters of the individual stars. Consequently it will influence the conclusions regarding the evolution of the stellar members, and thus also those of the cluster itself.

Besides Walker (1961), other authors placed many objects several magnitudes above the MS, e.g. Sagar \& Joshi (1979), Chini \& Wargau (1990) and Hillenbrand et al. (1993). However, Chini \& Wargau (1990) critically questioned whether these stars located far above the MS, are true PMS members. From their study they concluded that their 29 programme stars are in reality foreground objects. The problem of recognizing NGC 6611 field stars as true cluster members is already discussed in Thé et al. (1990). The results of a proper-motion survey and the minimum foreground extinction values are the tools for discriminating between cluster members and foreground objects. The results of these studies will be used for our programme stars together with new conclusions based on their location in the HRD of NGC 6611. Given the probable age of NGC 6611, PMS objects are expected to occur. Therefore it is unlikely that all PMS candidates are foreground stars.

The existence of PMS objects in NGC 6611 was shown more recently by Hillenbrand et al. (1993). They published a study based on $U B V$ and $J H K$ imaging, down to magnitude 15 , over the whole field of M 16. Placing the objects in HRDs, two cluster populations were identified: the more massive ones, $M_{\star}>9 M_{\odot}$, being in their post-zeroage main sequence (ZAMS) phase, and intermediate mass stars, $3 M_{\odot}<M_{\star}<8 M_{\odot}$, being in their pre-ZAMS phase. From their study the cluster age was determined to be probably less than $(2 \pm 1)$ Myr. Many objects were found to be located even further above the MS, meaning that they are just formed. In the $J H K$ images many objects were found to be embedded. This could mean that we are actually still observing recent or even on-going star formation, which indicates a significant age spread in the cluster population. This suggestion is supported by the detection of an evolved B2.5 I star of about $30 M_{\odot}$. High mass star formation must also haven taken place about 6 Myr ago. As most pre-ZAMS objects studied by Hillenbrand et al. (1993) do not exhibit a near-IR excess, 
which indicates the presence of an (accretion-)disk, the possible ages will put strong constraints on the disk lifetimes for intermediate mass stars.

It should be noted, however, that Hillenbrand et al. (1993) have used the non-individual approach for the different cluster members. They used colour-colour diagrams for de-reddening and for investigating the anomalous extinction. An overall $R_{V}$ value of 3.75 for the anomalous extinction was derived. Therefore, the results of Hillenbrand et al. (1993) are not conclusive.

A spectral study of 73 objects was done by Hillenbrand et al. (1993) and they detected several emission line objects. Several are showing properties as Herbig $\mathrm{Ae} / \mathrm{Be}(\mathrm{HAeBe})$ objects. Other stars have IR- and emission-line properties indicative of classical Ae/Be stars. These authors questioned whether part of the classical Ae/Be-group could actually be young, objects having a gaseous disk left-over from star formation.

In Thé et al. (1990) 52 out of our 89 programme stars with a high probability of membership in NGC 6611 were studied. The selected stars are the ones not recognized as PMS candidates. They confirmed the extreme youth of NGC 6611 and used the location of several massive stars in the HR-diagram to derive an age not much more than $510^{6}$ yrs. They also demonstrated many characteristics of NGC 6611, such as the above-mentioned points (a) and (b), and determined some other important parameters. The distance of $2.6 \pm 0.3 \mathrm{kpc}$ and the detection of individual extinction laws towards each object, rather than an average one for part of the whole cluster, are the most important ones. Intrinsic stellar parameters are then easily determined if fundamental observations, such as low-resolution spectroscopy and photometry, have been made.

In this study the PMS candidates will be investigated. The selected 49 stars are collected from those listed by Walker (1961), Sagar \& Joshi (1979), Chini \& Wargau (1990) and given in Thé et al. (1990). We have put no limits for the spectral type, such as the cut-off at A0 in the study of Hillenbrand et al. (1993). In this study we also applied the individual approach. It is more extensive and detailed than the initial PMS search made by de Winter \& Thé (1991) in NGC 6611. For all the programme stars low-resolution spectral and photometric observations have been made or collected from previous studies. The spectra and spectral energy distributions are studied and a spectral type and intrinsic magnitudes are derived. This information is used to determine the extinction law for individual objects as well as their astrophysical parameters. Together with the results of our previous study (Thé et al. 1990), we will study the HRD of NGC 6611, the probability of the detection of Herbig Ae/Be stars in our sample, the distribution of the extinction in the field of NGC 6611 and the membership of the programme stars. Finally we will test the $\mathrm{HAeBe}$ candidates on typical characteristics such as the presence of an infrared excess, variability and emission lines.

\section{Observations}

\subsection{Photometric observations}

As mentioned in the introduction we have used new observations as well as older data.

The observed Walraven $W U L B V$ photometric data are presented in Table 1. They were collected in August 1985 and August 1986 with a photometer attached to the $90 \mathrm{~cm}$ Dutch Light Collector stationed at ESO. These observations were made through a $16^{\prime \prime} .5$ diaphragm, and were calibrated using standard stars published by Lub \& Pel (1977) of which the data are updated.

Observations in $U B V R_{\mathrm{C}} I_{\mathrm{C}}$, taken during June 1983, July 1985 and August 1986, and JHKLM, made in July 1986, are listed in Tables 2 and 3. The August 1986 observations were all taken at relatively high airmass and are therefore of low quality. The equipment used for these observations is described in Thé et al. (1990).

The data from the literature are taken from:

1) Thé et al. (1990): for a number of selected PMScandidates the observations are listed and described in Thé et al. (1990). These observations include $U B V R_{\mathrm{C}} I_{\mathrm{C}}$ as well as $J H K L M$ data. In this paper different observing $U B V R_{\mathrm{C}} I_{\mathrm{C}}$ runs were compared and no systematic differences between photoelectric data sets were found.

2) Walker (1961): only the photographic observations, taken on June 1955 with the 100-inch reflector were used. The plates contain all stars brighter than about $V=16^{\mathrm{m}} \cdot 5$. Only in some cases did the data sets appear to be useful with respect to the photoelectric observations.

3) Sagar \& Joshi (1979): the observations were carried out between April 1977 and October 1978, with the $104 \mathrm{~cm}$ Sampurnanand reflector of the Uttar Pradesh State Observatory, India, employing standard $U B V$ filters of the Johnson system. A minimum of two sets of observations, on different nights, were taken for each star. The averaged $U B V$ values are taken for our purpose.

4) Chini \& Wargau (1990): Johnson $U B V R I$ data were observed with the $0.5 \mathrm{~m}$ telescope on Gamsberg in Namibia and with the $1.23 \mathrm{~m}$ telescope of the DSAZ on Calar Alto in Spain from 1978 to 1981. The JHKL data were taken with the ESO $1 \mathrm{~m}$ telescope on La Silla during August 1987 and with the SAAO $1.9 \mathrm{~m}$ telescope in Sutherland during July 1988 . Their programme stars were measured at least twice in both the $U B V R I$ and $J H K(L)$ Johnson photometric systems. Only the averaged values are given.

5) Hillenbrand et al. (1993): all measurements were made at the Kitt Peak National Observatory (KPNO). The photometric imaging data, taken in October 1990 using a $90 \mathrm{~cm}$ telescope, consists of several sets of overlapping $U B V$ optical images covering approximately $1600 \operatorname{arcmin}^{2}$ 
centered on the cluster. Furthermore mosaicked $J H K$ infrared images covering roughly the central $700 \mathrm{arcmin}^{2}$ of the cluster were made with the KPNO $1.3 \mathrm{~m}$.

All observations of our sample stars, as derived from above studies, are listed in Table 2. In this table several data sets are clearly not in agreement with each other, e.g. the $U B V$ data of $\mathrm{W} 494$. Besides observational errors, variability can be the cause. A discussion of useful data for each object will be given in Sects. 4 and 5. A discussion of all errors involved in the observations and the averaging procedures are given by Koulis (1993).

\subsection{Spectroscopy}

For most of our programme stars a low resolution $\left(172 \AA \mathrm{mm}^{-1}\right)$ spectrum was taken in July 1985 or July 1986 with the Image Dissector Scanner (IDS) attached to the Boller and Chivens spectrograph mounted on the ESO $1.52 \mathrm{~m}$ telescope. These spectra cover a spectral range from about 3700 to $7000 \AA$. Because the IDS data was incomplete and sometimes not well exposed, we made low dispersion $\left(2 \AA\right.$ pixel $\left.^{-1}\right)$ spectra for each programme star with the ESO $1.52 \mathrm{~m}$ telescope in June 1992, to which a Boller and Chivens spectrograph was attached. The detector is a high resolution Ford Aerospace $2048 \times 2048$ $\mathrm{CCD}$, with a pixel size of $15 \times 15 \mu \mathrm{m}$. The dispersion of $172 \AA \mathrm{mm}^{-1}$ produces a usable spectral range from 4200 to $7800 \AA$.

All the IDS and CCD spectra obtained for our programme stars are given in Koulis (1993). For reasons which will be discussed in Sect. 10, we present only the CCD spectra, see Fig. 1.

\section{Spectral classification}

A reliable spectral type for each programme star is of fundamental importance in order to obtain the accurate information about its extinction characteristics and, consequently, its location in an HR-diagram. In most previous studies only photometric spectral types were available. Such spectral types are only reliable when obtained at maximum brightness, as they can be influenced by anomalous extinction and variability. Furthermore, since photometric spectral types are not always unique, spectral observations are indispensable. However, because of the occurrence of spectral line changes in HAeBes (e.g. Grinin et al. 1994) the spectral type derived from the continuum is certainly welcome.

\subsection{Photometry: Two colour diagrams}

Two-Colour Diagrams (TCDs) for cluster members can be used to determine the foreground extinction, to describe a probable common extinction behaviour and to estimate photometric spectral types by dereddening the observed colours to intrinsic ones if an extinction law is assumed. In Thé et al. (1990) the foreground $E(B-V)$ for
NGC 6611 was determined to be at least $0{ }^{\mathrm{m}} 5$. We have seen from several studies that the extinction law for each star can be different. The use of a TCD to determine extinction laws for clusters like NGC 6611, as was done in Chini \& Wargau (1990), is therefore not reliable. We will use TCDs of the Johnson $U B V$ and the Walraven $U L B V$ data to estimate the spectral types of our programme stars and to see if our objects are lying at the foreground.

\subsubsection{The $B-V$ versus $U-B$ two colour diagram}

In Fig. 2 the TCD based on the $U B V$ data is given for our programme stars. In this figure relations for luminosity classes III and $\mathrm{V}$ are plotted using the intrinsic $(B-V)_{0}$ and $(U-B)_{0}$ colours listed in SchmidtKaler (1982). Furthermore, the reddening slope of $E(U-$ $B) / E(B-V)=0.72$ is indicated. This value corresponds to the normal extinction law, characterized by $R_{V}$ : $=$ $A_{V} / E(B-V)=3.1 \pm 0.1$. Such an extinction law has also been adopted for the foreground interstellar medium (Thé et al. 1990). By dereddening the observed $U B V$ data of a star along this line, the intersection with the intrinsic $U B V$ relations will give us the extinction values, $E(B-V)$ and $E(U-B)$. The intrinsic values are determined by: $(B-V)_{0}=(B-V)-E(B-V)$ and $(U-B)_{0}=(U-B)-E(U-B)$. Comparing those values to the ones listed in Schmidt-Kaler's (1982) Table 12a we can derive the photometric spectral type of the star. In this method a separation in luminosity class seems to be significant only for the coolest objects.

It should be noted that in the above procedure we have used $R_{V}=3.1$ for all stars, regardless of their true $R_{V}$ value. This is justified, because the reddening slope of 0.72 of the $U B V$ system is insensitive to anomalous extinction, expressed by the $R_{V}$ value. This can be shown from data of Steenman \& Thé (1989 and 1991). A spectral type derived from the TCD method is, therefore, quite reliable. For each photometric data set a spectral type was derived. The ones which are consistent were averaged, resulting in the $\langle U B V\rangle$ spectral type given in Table 4.

As can be seen from Fig. 2 the photometric spectral type can sometimes not be uniquely derived; there are two or three intersections with the ZAMS possible, resulting in several spectral types for a given star. We will remove this ambiguity using available spectra and the spectral energy distribution (SED) fits, which will be described below.

\subsubsection{The Walraven $[B-L]$ versus $[B-U]$ two colour diagram}

The Walraven data for our programme stars are listed in Table 1. Using these data we wish to deduce a photometric spectral type for each programme star, which can not be simply done as for the $U B V$ data. The Walraven two-colour diagram is based on the intrinsic colours $[B-L]$ versus $[B-U]$. The observed colours $(B-U)$ and 


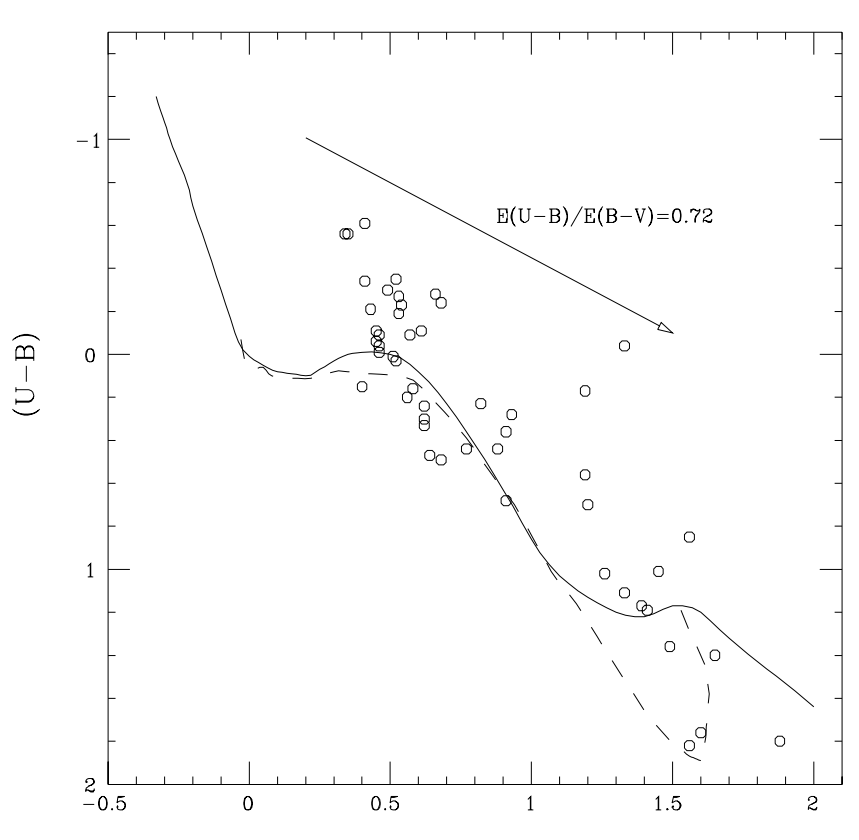

$(\mathrm{B}-\mathrm{V})$

Fig. 2. The $U-B, B-V$ diagram for our stars in NGC 6611. The intrinsic luminosity class V relation (solid line) and the one for class III (dashed line) of Schmidt-Kaler (1982) are drawn

$(B-L)$ can be transformed into reddening-free ones using the following relations (Brand \& Wouterloot 1988):

$[B-U]=(B-U)-0.61 \times(V-B)$

$[B-L]=(B-L)-0.39 \times(V-B)$.

The coefficients in these equations are the slopes of the reddening lines in the corresponding colour-colour diagrams, and depend on the reddening law (the normal extinction law has been assumed, i.e. $R_{V}=A_{V} / E(B-$ $V)_{\mathrm{W}}=3.2$, Schmidt-Kaler 1982) and the properties of the passbands. Note that $R_{V}=3.2$ for the Walraven photometric system is equivalent to $R_{V}=3.1$ for the $U B V R I$ Johnson photometric system.

The derivation of $\log T_{\text {eff }}$ and $\log g$ from the observed reddening-free colours is done through linear interpolation in the reddening free diagrams (Fig. 3a) of Brand \& Wouterloot (1988). Then using Table 3 of Schmidt-Kaler (1982), we find for each star its spectral type listed in Table 4.

Note that the Walraven data are not available for all programme stars (see Table 1 ). Note also that several faint and late type stars (for which this method is not appropriate), W266, W349, W402, W494, W525, W611 and W617, are completely out of the diagram. A few other stars (W213, W299, W469, W556 and W605) lie too far away from the intrinsic $\left(T_{\text {eff }}, \log g\right)$ models. For these stars no reliable photometric spectral types can be deduced.

\subsection{Spectroscopy: Visual-red low resolution spectra}

For the spectral classification of both the IDS and the CCD spectra of our programme stars we used the spectral catalogue of Jacoby et al. (1984), which contains spectra for classes O-M and luminosity classes V, III and I, and Sect. 4 of Schmidt-Kaler (1982) in which the main spectral descriptions are given for the MK classification.

A complete description of the main criteria that were applied to classify the available spectra of our programme stars is presented by Koulis (1993). The reduced IDS spectra are presented in Appendix A of Koulis (1993). The reduced CCD spectra are given in Fig. 1. All deduced spectral types are collected in Table 4.

\section{Spectral energy distribution}

Using the stellar spectral types and the photometric observations made through the different pass-bands, we can draw spectral energy distributions (SEDs). By fitting the SED to a Kurucz (1991) model, with solar abundances, appropriate to the MK-type of the star, we obtain information concerning the correct spectral classification, the extinction at the different wavelengths and a possible IRexcess. Below we will discuss briefly the fitting procedure, the selection of the photometric data and the estimation of the Kurucz parameters.

In most cases the value $R_{V}=3.1$ can be used to correct for the extinction due to the interstellar medium. However, we have encountered objects which, after correction with $R_{V}=3.1$, show SEDs with a small near-infrared excess. Taking into account previous studies of NGC 6611 and the characteristics of PMS stars, we assume that the existence of an infrared excess is caused, either by anomalous extinction towards the star, or, when no extinction law can be fitted easily, by the presence of circumstellar dust.

The observed magnitudes are first corrected assuming a normal interstellar extinction law. The resulting extinction-free SED is then compared with the theoretical SED model of Kurucz (1991), appropriate to the MK type of the star. When an infrared excess remains, an $R_{V}$ value of one tenth higher is applied. If the infrared excess still exists, the same procedure is repeated until a best fit between the observed extinction-free SED and the Kurucz model is obtained according to the $\chi^{2}$-test. The $R_{V}$ value of the best fit is then adopted. The extinction laws used in this procedure are those of Steenman \& Thé (1991).

The $\chi^{2}$-test is applied to the difference between the extinction-free SED and the Kurucz model for all pass bands with wavelengths between the $\mathrm{B}$ passband at $0.434 \mu \mathrm{m}$ and the near-IR $K$ passband at $2.2 \mu \mathrm{m}$. The errors in the observed data (Koulis 1993) are taken into account in the $\chi^{2}$-test procedure. For a better accuracy, especially around the Balmer jump, we have integrated over the response curve of each filter instead of using monochromatic fluxes. 


\subsection{Selection of the photometric data}

From Tables 1-3 we see that in most cases multiple sets of photometric data are available. In addition, several stars display the typical HAeBe characteristic of photometric variability. The ones with a visual variability range significantly more than 0.2 are marked with a star in Table 7.

Because of the large amount of data that had slowly accumulated and because the differences in most of the $U B V(R I)_{\mathrm{J}, \mathrm{C}}$, Walraven and $J H K L M$ data sets are only small, we have decided to average the data sets that appeared to be compatible, preferably with the highest intensity to acquire data at maximum light. The agreements of so many data sets indicate that we are working with reliable photometric observations. The increase of accuracy in the various photometric colours implies a more accurate determination of the colour excess, the photometric spectral type, and consequently the (possibly anomalous) extinction.

The photometric data were selected using the following criteria:

For the Walraven data in the visual we have first calculated the corresponding Johnson visual magnitudes $V_{\mathrm{J}}$, using the relation (Pel 1987):

$V_{\mathrm{J}}=6.886-2.5 \times\left[V_{\mathrm{W}}+0.033 \times(V-B)_{\mathrm{W}}\right]$.

When a $V_{\mathrm{J}}$ agrees with the $V$ in the $U B V(R I)_{\mathrm{J}, \mathrm{C}}$ system, it is considered eligible for further use.

We used a maximum of 5 sets of $U B V$ data per star. For some stars we had only one set of Johnson $R_{\mathrm{J}} I_{\mathrm{J}}$ and/or Cousins $R_{\mathrm{C}} I_{\mathrm{C}}$ as noted in Table 2. Of the available $U B V R I$ data sets we only used the ones that are in mutual agreement, and for which the relative differences of the colours are minimal. A similar procedure was applied to the $J H K(L)$ data sets, but with 3 data sets per star at most.

Before averaging the selected data sets used for the SEDs, we must note that, when averaging data of different authors, i.e. taken during different observational periods with different telescopes, different sets of filters etc., one has to take into account the systematic differences that may arise between the various observing runs. Therefore we first must correct the data from all authors with respect to our data, references 1a-c in Table 2, by adding an average systematic difference. The systematic differences, as given in Table 5, are taken from Thé et al. (1990) and Hillenbrand (1993), except for Chini \& Wargau's data for which we calculated the correction ourselves. We also estimated the average systematic differences for the $J H K(L)$ colours. The Walraven and $R_{\mathrm{C}} I_{\mathrm{C}}$ data did not need a correction as they were made in one system. The same applies to the $R_{\mathrm{J}} I_{\mathrm{J}}$ data of Chini \& Wargau (1990).

All corrected and averaged magnitudes and colours are given in Appendix C of Koulis (1993). Table 6 lists the accordingly derived photometric data sets which were used to construct the SED of each star.

\subsection{Estimate of the Kurucz parameters}

To fit the selected photometry to a Kurucz (1991) model, we first need to consider the best $T_{\mathrm{eff}}, E(B-V)$ and $\log$ $g$ for each object in order to select its appropriate model.

In Table 4 there are a number of spectral types that have been derived from the photometric and spectroscopic data of each star which were already in close agreement with each other. As we want one final spectral type for each star we averaged those spectral types, giving a weight of 2 to the averaged spectral type deduced spectroscopically and 1 to the averaged photometric spectral type.

Although the spectral types and therefore the $T_{\text {eff }}$ 's are accurately known, an SED fit of the Kurucz model to the selected photometry can reveal some small modifications needed to find the correct Kurucz model. In these cases we determined a more exact $T_{\text {eff }}$ in an iterative way.

By this final check of the stellar spectral type we determined the $(B-V)_{0}$ value from Schmidt-Kaler (1989). The $E(B-V)$ is then the difference between this $(B-V)_{0}$ value and the $(B-V)$ used in the SED.

Note that a wrong predetermination, á priori or iteratively, of the spectral type or $T_{\text {eff }}$, could make a foreground object seem to be a cluster member and vice versa. In NGC 6611, cluster members should have $E(B-V)>$ 0.50 .

A third but also very important parameter is the star's luminosity class. Although, varying the spectral type within a luminosity class will not alter the $\log g$ values very much, changing the $\log g$ implicitly affects the $(B-V)_{0}$ and therefore the $E(B-V)$ values of the star. This parameter can, therefore, affect the cluster membership probability of a star in two different ways: (1) because it influences its $E(B-V)$, it could drop below 0.50 , and (2) the star's position in the HRD.

\subsection{The fits to the SEDs}

Adopting the average of the selected photometric data, listed in Table 6, the average spectral type, listed in Table 4 , and the above mentioned determination of the $E(B-V)$ value, we are able to fit the SED for each programme star to a Kurucz model SED. Because of the unknown luminosity class for most objects we adopted $\log g=4.0$ (luminosity class IV or V). The observed magnitudes are first corrected assuming a normal extinction law $\left(R_{V}=3.1\right)$. The extinction-free SED is then plotted. A comparison is finally made with the theoretical SED model of Kurucz (1991), appropriate to the MK type of the star.

Although in many cases the SED can be satisfactorily fitted, several cannot. We made the next modifications to 
Table 5. Systematic differences of stellar $U B V$ and $J H K(L)$ data in NGC 6611 for the observations taken in our programme minus those of other authors. $n$ indicates the number of available datasets

\begin{tabular}{lrrrr}
\hline References & \multicolumn{1}{c}{$B$} & \multicolumn{1}{c}{$B-V$} & \multicolumn{1}{c}{$U-B$} & $n$ \\
\hline Walker (1961) pg & $-0.02 \pm 0.12$ & $-0.07 \pm 0.14$ & $0.06 \pm 0.14$ & \\
Sagar \& Joshi (1979) & $-0.02 \pm 0.13$ & $-0.01 \pm 0.07$ & $-0.03 \pm 0.07$ & 40 \\
Chini \& Wargau (1990) & $0.02 \pm 0.05$ & $0.02 \pm 0.03$ & $-0.03 \pm 0.09$ & 9 \\
Hillenbrand et al. (1993) & $-0.02 \pm 0.04$ & $0.02 \pm 0.01$ & $-0.014 \pm 0.01$ & 80 \\
\hline
\end{tabular}

\begin{tabular}{lccccc}
\hline References & $J$ & $H$ & $K$ & $L$ & $n$ \\
\hline Chini \& Wargau (1990) & $0.00 \pm 0.07$ & $-0.19 \pm 0.10$ & $-0.07 \pm 0.12$ & $-0.09 \pm 0.01$ & 10 \\
Hillenbrand et al. (1993) & $0.04 \pm 0.07$ & $0.03 \pm 0.07$ & $-0.04 \pm 0.13$ & & 23 \\
\hline
\end{tabular}

improve the latter fits:

1. In case the $W U L B V$ and $U B V R I$ photometry did not fit well, especially around the Balmer jump, we altered the temperature $T_{\text {eff }}$ of the star.

2 . If the fit was still not adequate and we noted a depletion in the $J H K(L M)$ bands, we changed the luminosity class to III, II or I, which means that we lower the values of $\log g$. Varying $\log g$ of Kurucz models will only cause significant changes for late, G-M, type objects.

3. In those cases where the SED fit still show a discrepancy in the near-IR we applied the $R_{V}$-fitting procedure in order to test the presence of anomalous extinction.

4. Some stars show a large IR-excess that can not be corrected with any reasonable anomalous extinction law. In these cases we fit a Planckian curve. If we assume circumstellar dust to be responsible for the excess, the wavelength at which the fitted Planckian-curve reaches its maximum indicates the temperature of the dust (Sect. 5.3). Note that for very high $R_{V}$ values a too strong correction of the extinction in the blue and nearUV pass-bands will cause a depletion of the for extinction corrected observed SED compared to the Kurucz model fitted to the optical datapoints.

For some stars, we have to discard certain data sets which are not in mutual agreement, and reduce the reliability of the SED fitting results:

For W213(1) and W494 the WULBV data;

For W525 we only use the Walraven passbands $W$ and $U$; For W617 we discard the Walraven $W$ band and the $U B V$ reference $1 \mathrm{~b}$ data;

For W213(2) and W267 we discard the $R_{\mathrm{C}}$ and $I_{\mathrm{C}}$ data; For W245 both the $R_{\mathrm{J}} I_{\mathrm{J}}$ and $R_{\mathrm{C}} I_{\mathrm{C}}$ have to be omitted;

For W400 we discard the $R_{\mathrm{J}} I_{\mathrm{J}}$ and the Walker (1961) $U$ because it differed too much from other $U$ observations; For W349 and W402 $I_{\mathrm{J}}$ have to be ignored;

For $\mathrm{W} 455 I_{\mathrm{C}}$ is not used;

For W300 and W406 we do not use the JHK observations as they appeared to lie far above or below the best-fit Kurucz model.

All the resulting plots are presented in Fig. 3. The final astrophysical parameters as derived and checked by the SED fits are given in Table 7.

\section{Analysis of the SED fits}

This section can be found in the electronic version at http://www.ed-phys.fr

\section{The Hertzsprung-Russell diagram of NGC 6611}

As discussed in the previous sections, we finally obtained for each programme star a good SED fit including accurate estimates of the stellar astrophysical parameters, such as $E(B-V), T_{\text {eff }}, R_{V}$ and $(B-V)_{0}$ values, which are collected in Table 7 . With the intrinsic stellar parameters $V_{0}$ and $(B-V)_{0}$, we can construct an HRD. $V_{0}$ is simply estimated from the difference between the observed $V$, for which we used the values from the best SED fits, and $A_{V}$, with $A_{V}=E(B-V) \times R_{V}$.

As discussed in Sect. 5, for a few objects we have multiple possibilities in the SED fit. They will be analyzed as being separate objects. With the data of the remaining 52 objects we construct an HRD in Fig. 4. In this figure we have discriminated to $R_{V}$ values by plotting different symbols: a cross for stars with $R_{V}=3.1$, a square for stars with $R_{V}>3.1$ and circles for objects with unknown extinction characteristics: the objects which are denoted by sh.

Figure 4 also contains two stellar birthlines of Palla \& Stahler (1990 and 1992), which is the boundary after which stars become optically visible when evolving towards the main sequence. The upper one is for an accretion rate on the protostar of $\dot{M}_{\mathrm{acc}}=10^{-4} M_{\odot} \mathrm{yr}^{-1}$ and the lower one for $\dot{M}_{\text {acc }}=10^{-5} M_{\odot} \mathrm{yr}^{-1}$. We have also drawn the Zero Age Main Sequence of Hillenbrand et al. (1993). The birthlines and ZAMS are recalculated from the published HRDs to our observational HRD by adopting a distance of $2.6 \mathrm{kpc}$ and a luminosity class III. 
Table 7. Physical data of stars in NGC 6611 . Objects indicated by a star $\left.{ }^{\star}\right)$ show a visual variability range significantly larger than 0.2 . In the last column the probability of membership is given by the value $P$ from Thé et al. (1990) and references therein, the values of Kamp (1974) are given between parentheses. Note that several spectral types have been adjusted to their SED fits. The luminosities given in Col. 11 are those obtained from $M_{\mathrm{bol}}$, whereas those in Col. 12 are obtained from an integration over the SED (see text)

\begin{tabular}{|c|c|c|c|c|c|c|c|c|c|c|c|c|}
\hline Name & Sp.T. & $R_{V}$ & $E(B-V)$ & $A_{V}$ & $V_{0}$ & $(B-V)_{0}$ & $\log T_{\text {eff }}$ & $M_{V}$ & $M_{\text {bol }}$ & $\log L_{\star} / L_{\odot}$ & $\left(\log L_{\star} / L_{\odot}\right)_{\mathrm{SED}}$ & $P[\%]$ \\
\hline W103 & B9 & 3.10 & $0^{\mathrm{m}} 43$ & $1{ }^{\mathrm{m}} 33$ & $11^{\mathrm{m}} 15$ & $-0 \mathrm{~m}^{\mathrm{m}} 03$ & 4.022 & $-00^{\mathrm{m}} 95$ & $-1^{\mathrm{m}} 46$ & 2.44 & 2.46 & $90,(0)$ \\
\hline W188 & B0 & 3.30 & $1 \mathrm{~m}^{\mathrm{m}} 63$ & $5^{\mathrm{m}} 38$ & $7^{\mathrm{m}} 72$ & $-0^{\mathrm{m}} 30$ & 4.486 & $-4^{\mathrm{m}} 38$ & $-7^{\mathrm{m}} 54$ & 4.87 & 4.86 & 87 \\
\hline W202 & B3 & 3.60 & $1^{\mathrm{m}} 13$ & $4^{\mathrm{m}} 07$ & $10 \mathrm{~m}_{30}$ & $-0 \mathrm{~m}_{20}$ & 4.271 & $-1 \mathrm{~m}^{\mathrm{m}} 80$ & $-3^{\mathrm{m}} 74$ & 3.35 & 3.34 & 0 \\
\hline W207 & B1 & 3.60 & $0{ }^{\mathrm{m}} 78$ & $2^{\mathrm{m}} 81$ & $9 \mathrm{~m}^{\mathrm{m}} 23$ & $-0 \mathrm{~m}_{26}$ & 4.405 & $-2^{\mathrm{m}} 87$ & $-5^{\mathrm{m}} 57$ & 4.08 & 4.07 & $88,(92)$ \\
\hline $\mathrm{W} 213(1)^{\star}$ & A7 & 3.40 & $1 \mathrm{~m}_{27}^{\mathrm{m}}$ & $4^{\mathrm{m}} 32$ & $9^{\mathrm{m}} 66$ & $0^{\mathrm{m}} 20$ & 3.895 & $-2^{\mathrm{m}} 44$ & $-2^{\mathrm{m}_{5}}$ & 2.88 & 2.91 & \\
\hline $\mathrm{W} 213(2)^{\star}$ & F9 & 3.40 & $1 \mathrm{~m}^{\mathrm{m}} 01$ & $3^{\mathrm{m}} 43$ & $10 \mathrm{~m}^{\mathrm{m}} 70$ & $0^{\mathrm{m}} 55$ & 3.786 & $-1 \mathrm{~m}^{\mathrm{m}} 40$ & $-1 \mathrm{~m}_{57}$ & 2.48 & 2.52 & \\
\hline $\mathrm{W} 232^{\star}$ & F8 & 4.00 & $0^{\mathrm{m}} 39$ & $1{ }^{\mathrm{m}} 56$ & $12^{\mathrm{m}} 76$ & $0^{m} 52$ & 3.792 & $0 \mathrm{~m}^{\mathrm{m}} 66$ & $0^{\mathrm{m}} 50$ & 1.66 & 1.57 & \\
\hline W240 & B8 & 3.10 & $0{ }^{\mathrm{m}} 67$ & $2^{\mathrm{m}} 08$ & $12^{\mathrm{m}} 47$ & $-0 .{ }^{\mathrm{m}} 11$ & 4.077 & $0^{\mathrm{m}} 37$ & $-0 . m^{m} 43$ & 2.03 & 2.01 & 0 \\
\hline $\mathrm{W} 245 \mathrm{sh}^{\star}$ & $\mathrm{B} 6$ & 3.10 & $0^{\mathrm{m}} 92$ & $2 \cdot 85$ & $10^{\mathrm{m}} 77$ & $-0 \mathrm{~m}^{\mathrm{m}} 15$ & 4.146 & $-1^{\mathrm{m}} 33$ & $-2^{m} 54$ & 2.87 & 2.88 & 0 \\
\hline W262sh & B7 & 3.10 & $0{ }^{m} 65$ & $2^{\mathrm{m}} 02$ & $11^{\mathrm{m}} 90$ & $-00^{\mathrm{m}} 13$ & 4.115 & $-0^{\mathrm{m}} 19$ & $-1^{\mathrm{m}_{21}}$ & 2.34 & 2.33 & 57 \\
\hline $\mathrm{W} 266 \mathrm{sh}^{\star}$ & F8 & 3.10 & $0^{\mathrm{m}} 68$ & $2^{\mathrm{m}} 11$ & $12^{\mathrm{m}_{2}}$ & $0^{\mathrm{m}} 52$ & 3.792 & $0^{\mathrm{m}} 13$ & $-0 \mathrm{~m}^{\mathrm{m}} 03$ & 1.87 & 1.89 & \\
\hline W267 & $\mathrm{B} 2$ & 3.10 & $0{ }^{m} 78$ & $2 m^{m}$ & $10^{\mathrm{m}} 71$ & $-0{ }^{\mathrm{m}} 24$ & 4.342 & $-1 \mathrm{~m} 39$ & $-3^{m} 74$ & 3.35 & 3.35 & 92 \\
\hline W273* & A0 & 4.80 & $0{ }^{\mathrm{m}} 64$ & $3{ }^{\mathrm{m}} 07$ & $11^{\mathrm{m}} 11$ & $-0 \mathrm{~m}^{\mathrm{m}} 02$ & 3.978 & $-00^{m} 99$ & $-1 \mathrm{~m}_{29}$ & 2.37 & 2.34 & 94 \\
\hline W273sh* & A0 & 3.10 & $0^{\mathrm{m}} 64$ & $1^{\mathrm{m}} 98$ & $12 \mathrm{~m}_{20}$ & $-0{ }^{\mathrm{m}} 02$ & 3.978 & $0^{\mathrm{m}} 10$ & $-0 m_{20}$ & 1.94 & 2.00 & 94 \\
\hline W276(1) & B5 & 3.40 & $00^{\mathrm{m}} 74$ & $2^{\mathrm{m}} 52$ & $11 \mathrm{~m}_{21}$ & $-0 \mathrm{~m}^{\mathrm{m}} 17$ & 4.188 & $0{ }^{\mathrm{m}} 89$ & $-2^{\mathrm{m}} 35$ & 2.80 & 2.76 & 89 \\
\hline W290 & $\mathrm{B} 2$ & 3.80 & $00^{\mathrm{m}} 65$ & $2^{\mathrm{m}} 47$ & $9 \mathrm{~m}^{\mathrm{m}} 66$ & $-0 \mathrm{~m}_{24}$ & 4.342 & $-2^{\mathrm{m}} 44$ & $-4^{\mathrm{m}} 79$ & 3.77 & 3.66 & $93,(18)$ \\
\hline W297 & $\mathrm{B} 1$ & 3.60 & $0^{\mathrm{m}} 94$ & $3^{\mathrm{m}} 38$ & $9^{m} 49$ & $-0 \mathrm{~m}_{26}$ & 4.405 & $-2^{\mathrm{m}} 61$ & $-5^{\mathrm{m}} 77$ & 4.16 & 3.92 & $0,(0)$ \\
\hline W299(1) & $\mathrm{B} 6$ & 3.80 & $0{ }^{\mathrm{m}} 83$ & $3^{\mathrm{m}} 15$ & $10^{\mathrm{m}} 99$ & $-0 m_{15}^{m}$ & 4.146 & $-1^{\mathrm{m}} 11$ & $-2^{\mathrm{m}} 32$ & 2.78 & 2.72 & 0 \\
\hline W299(1) sh & $\mathrm{B} 6$ & 3.10 & $0^{\mathrm{m}} 83$ & $2^{\mathrm{m}} 57$ & $11^{\mathrm{m}_{57}}$ & $-0 \mathrm{~m}_{15}^{\mathrm{m}}$ & 4.146 & $-0^{\mathrm{m}} 53$ & $-1^{\mathrm{m}} 74$ & 2.55 & 2.55 & 0 \\
\hline W300 & $\mathrm{B} 2$ & 3.10 & $0^{\mathrm{m}} 77$ & $2^{\mathrm{m}} 39$ & $10 \mathrm{~m}_{27}$ & $-0 \mathrm{~m}_{24}$ & 4.342 & $-1 \mathrm{~m}^{\mathrm{m}} 83$ & $-4 \mathrm{~m}^{\mathrm{m}} 18$ & 3.53 & 3.55 & (0) \\
\hline W306 & B2 & 3.40 & $0{ }^{\mathrm{m}} 90$ & $3 \mathrm{~m}^{\mathrm{m}} 06$ & $9 \mathrm{~m}^{\mathrm{m}} 68$ & $-0 \mathrm{~m}_{24}^{\mathrm{m}}$ & 4.342 & $-2^{\mathrm{m}} 42$ & $-4^{\mathrm{m}} 77$ & 3.76 & 3.75 & $89,(82)$ \\
\hline W313* & B4 & 3.40 & $0{ }^{\mathrm{m}} 72$ & $2^{\mathrm{m}} 43$ & $10 . \mathrm{m}_{49}$ & $-0 .{ }^{\mathrm{m}} 19$ & 4.230 & $-1 . \mathrm{m}^{\mathrm{m}} 61$ & $-3 \cdot{ }^{\mathrm{m}} 31$ & 3.18 & 3.12 & 81 \\
\hline W336(2) & B3 & 3.50 & $0{ }^{\mathrm{m}} 69$ & $2^{\mathrm{m}} \cdot 42$ & $10 \cdot 85$ & $-0{ }^{\mathrm{m}} 20$ & 4.271 & $-1 \mathrm{~m}^{\mathrm{m}} 24$ & $-3 \cdot{ }^{\mathrm{m}} 18$ & 3.13 & 3.11 & 87 \\
\hline W339(2) & B3 & 3.90 & $1{ }^{\mathrm{m}} 08$ & $4 \mathrm{~m}^{\mathrm{m}}$ & $90^{m} 5$ & $-0^{m} 20$ & 4.271 & $-2^{m} 55$ & $-4^{\mathrm{m}} 49$ & 3.65 & 3.62 & \\
\hline W339(2)sh & B3 & 3.10 & $1 \mathrm{~m}^{\mathrm{m}} 08$ & $3^{\mathrm{m}} 35$ & $10^{\mathrm{m}} 41$ & $-0^{\mathrm{m}} \mathrm{m}_{20}$ & 4.271 & $-1^{\mathrm{m}} 69$ & $-3^{\mathrm{m}} 63$ & 3.31 & 3.32 & \\
\hline W349 & G8 I & 3.10 & $0{ }^{\mathrm{m}} 31$ & $0{ }^{\mathrm{m}} 96$ & $10 \mathrm{~m}^{\mathrm{m}} 66$ & $1{ }^{\mathrm{m}} 14$ & 3.663 & $-1^{\mathrm{m}} 44$ & $-1^{\mathrm{m}} 86$ & 2.60 & 2.98 & $90,(0)$ \\
\hline W $374^{\star}$ & $\mathrm{F} 2$ & 4.40 & $0^{\mathrm{m}} 56$ & $2^{\mathrm{m}} 46$ & $10^{\mathrm{m}} 93$ & $0^{\mathrm{m}} 35$ & 3.838 & $-1 \mathrm{~m}^{\mathrm{m}} 17$ & $-1 \mathrm{~m}_{28}$ & 2.37 & 2.31 & 0 \\
\hline W374 $\mathrm{sh}^{\star}$ & $\mathrm{F} 2$ & 3.10 & $0 \mathrm{~m}_{56}$ & $1 \mathrm{~m}^{\mathrm{m}} 74$ & $11^{\mathrm{m}} 65$ & $00^{\mathrm{m}} 35$ & 3.838 & $-0{ }^{\mathrm{m}} 45$ & $-0 \mathrm{~m}_{56}$ & 2.08 & 2.08 & 0 \\
\hline W388* & B6 & 4.00 & $0{ }^{m} 60$ & $2^{m} 40$ & $11 \mathrm{~m}_{27}$ & $-0^{m_{15}}$ & 4.146 & $-0{ }^{m} 83$ & $-2{ }^{m} 04$ & 2.67 & 2.61 & 91 \\
\hline W396(a) & F9 & 3.50 & $0 \mathrm{~m}^{\mathrm{m}} 64$ & $2^{\mathrm{m}} 24$ & $11^{\mathrm{m}} 74$ & $0^{\mathrm{m}} 55$ & 3.786 & $-0 \mathrm{~m}^{\mathrm{m}} 36$ & $-0 \mathrm{~m}_{53}$ & 2.07 & 2.08 & 0 \\
\hline W396(b) & G2 & 3.70 & $0^{\mathrm{m}} 56$ & $2{ }^{\mathrm{m}} 07$ & $11^{\mathrm{m}} 91$ & $0^{\mathrm{m}} 63$ & 3.768 & $-0^{\mathrm{m}} 19$ & $-00^{\mathrm{m}} 39$ & 2.01 & 2.03 & 0 \\
\hline W400 & B8 8 & 4.00 & $0^{\mathrm{m}} 73$ & $2^{\mathrm{m}} 92$ & $9 \mathrm{~m}^{\mathrm{m}} 92$ & $-0{ }^{\mathrm{m}} 11$ & 4.077 & $-2{ }^{m} 18$ & $-2^{m} 98$ & 3.05 & 3.01 & $93,(0)$ \\
\hline W402 & G9 III & 3.10 & $0^{\mathrm{m}} 51$ & $1^{\mathrm{m}} 58$ & $9^{m} 92$ & $0{ }^{\mathrm{m}} 97$ & 3.683 & $-2^{\mathrm{m}} 18$ & $-2^{\mathrm{m}} 64$ & 2.91 & 2.91 & $64,(0)$ \\
\hline W406 & K0 III & 3.10 & $0{ }^{\mathrm{m}} 88$ & $2^{\mathrm{m}} 73$ & $8^{\mathrm{m}} 96$ & $1{ }^{\mathrm{m}} 00$ & 3.676 & $-3^{\mathrm{m}} 14$ & $-3 \mathrm{~m}^{\mathrm{m}} 64$ & 3.31 & 3.33 & $0,(0)$ \\
\hline W411 & K5 III & 3.10 & $0^{\mathrm{m}} 10$ & $0{ }^{\mathrm{m}} 31$ & $11^{\mathrm{m}} 79$ & $1 \mathrm{~m}^{\mathrm{m}} 50$ & 3.596 & $-0{ }^{\mathrm{m}} 31$ & $-1 \mathrm{~m}^{\mathrm{m}} 33$ & 2.39 & 2.49 & $66,(85)$ \\
\hline W440(1) & G9 & 3.10 & $00^{\mathrm{m}} 61$ & $1 \mathrm{~m}^{\mathrm{m}} 89$ & $10 \mathrm{~m}_{35}$ & $0^{\mathrm{m}} 78$ & 3.733 & $-1^{\mathrm{m}} 75$ & $-2^{\mathrm{m}} 11$ & 2.70 & 2.65 & $0,(0)$ \\
\hline W455 & B7 & 3.10 & $0^{\mathrm{m}} 75$ & $2^{\mathrm{m}} 33$ & $9 \mathrm{~m}^{\mathrm{m}} 74$ & $-0 .{ }^{\mathrm{m}} 13$ & 4.115 & $-2^{\mathrm{m}} 36$ & $-3^{\mathrm{m}} 38$ & 3.21 & 3.17 & $7,(0)$ \\
\hline W469 & B2 & 3.40 & $0 \mathrm{~m}^{\mathrm{m}} 65$ & $2^{\mathrm{m}} 21$ & $8^{\mathrm{m}} 48$ & $-0 \mathrm{~m}_{24}$ & 4.342 & $-3^{\mathrm{m}} 62$ & $-5^{\mathrm{m}} 97$ & 4.24 & 4.22 & $94,(91)$ \\
\hline W483 & B 4.5 & 3.10 & $00^{\mathrm{m}} 61$ & $1^{\mathrm{m}} 88$ & $9 \mathrm{~m}^{\mathrm{m}} 07$ & $-0 .{ }^{m} 18$ & 4.209 & $-3{ }^{\mathrm{m}} 02$ & $-4^{\mathrm{m}} 60$ & 3.70 & 3.67 & $93,(93)$ \\
\hline W484 & B7 & 3.10 & $00^{\mathrm{m}} 64$ & $1^{\mathrm{m}} 98$ & $10 \mathrm{~m}^{\mathrm{m}} 42$ & $-0 .{ }^{m} 13$ & 4.115 & $-1 \mathrm{~m}^{\mathrm{m}} 68$ & $-2^{\mathrm{m}} 70$ & 2.94 & 2.96 & $94,(0)$ \\
\hline W489 & B7 & 3.30 & $0^{\mathrm{m}} 59$ & $1{ }^{\mathrm{m}} 95$ & $9{ }^{m} 60$ & $-0 \mathrm{~m}_{13}^{\mathrm{m}}$ & 4.115 & $-2^{m^{m}} 50$ & $-3^{\mathrm{m}_{52}}$ & 3.26 & 3.23 & $93,(91)$ \\
\hline $\mathrm{W} 494 \mathrm{sh}^{\star}$ & B6 & 3.10 & $0^{\mathrm{m}} 73$ & $2^{\mathrm{m}} 26$ & $11^{\mathrm{m}_{53}}$ & $-0{ }^{\mathrm{m}} 15$ & 4.146 & $-0^{\mathrm{m}} 57$ & $-1^{\mathrm{m}} 78$ & 2.57 & 2.59 & 1 \\
\hline W500* & B6 6 & 3.10 & $0 \mathrm{~m} 60$ & $1 \mathrm{~m}^{\mathrm{m}} 86$ & $99^{\mathrm{m}} 39$ & $-0{ }^{\mathrm{m}} 15$ & 4.146 & $-2^{m} 71$ & $-33_{92}$ & 3.42 & 3.42 & $94,(82)$ \\
\hline W504 & B5 & 3.10 & $0 m_{56}$ & $1 \mathrm{~m} 74$ & $11 \mathrm{~m}_{05}$ & $-0 \mathrm{~m}_{10}$ & 4.133 & $-1 \mathrm{~m} 05$ & $-2 \mathrm{~m} 00$ & 2.66 & 2.76 & $92,(0)$ \\
\hline W520 & B5 & 3.10 & $0 \mathrm{~m}^{\mathrm{m}} 63$ & $1 \mathrm{~m}_{95}^{\mathrm{m}}$ & $9{ }^{\mathrm{m}} 70$ & $-0 .{ }^{\mathrm{m}} 17$ & 4.188 & $-2 \mathrm{~m} 40$ & $-3 \mathrm{~m}^{\mathrm{m}} 86$ & 3.40 & 3.36 & $94,(7)$ \\
\hline W525* & K5 III & 3.10 & $0^{\mathrm{m}} 33$ & $1 \mathrm{~m}^{\mathrm{m}} 02$ & $12 \mathrm{~m}^{\mathrm{m}} 03$ & $1 \mathrm{~m}_{50}$ & 3.596 & $-0 \mathrm{~m}^{\mathrm{m}} 07$ & $-1 \mathrm{~m} 09$ & 2.29 & 2.26 & (75) \\
\hline W534* & K2 III & 3.10 & $0^{m} 25$ & $0^{\mathrm{m}} 78$ & $12 \mathrm{~m}^{\mathrm{m}} 08$ & $1^{\mathrm{m}} 16$ & 3.646 & $-00^{\mathrm{m}} 02$ & $-00^{\mathrm{m}} 63$ & 2.11 & 2.15 & $0,(88)$ \\
\hline W536 & $\mathrm{B} 1$ & 3.50 & $0^{\mathrm{m}} 60$ & $2^{\mathrm{m}} 10$ & $9^{\mathrm{m}} 32$ & $-0 \mathrm{~m}_{26}$ & 4.405 & $-2^{\mathrm{m}} 78$ & $-5^{\mathrm{m}} 48$ & 4.05 & 4.01 & $94,(92)$ \\
\hline W556 & $\mathrm{B} 2$ & 3.30 & $1^{\mathrm{m}} 43$ & $4^{\mathrm{m}} 72$ & $5{ }^{\mathrm{m}} 26$ & $-0 \mathrm{~m}_{24}$ & 4.342 & $-6{ }^{\mathrm{m}} 84$ & $-9^{\mathrm{m}} 19$ & 5.53 & 5.53 & $93,(43)$ \\
\hline W559 & B5.5 & 3.40 & $0^{\mathrm{m}} 75$ & $2^{\mathrm{m}} 55$ & $9{ }^{\mathrm{m}} 01$ & $-0 \mathrm{~m}_{15}^{\mathrm{m}}$ & 4.167 & $-3^{\mathrm{m}} 09$ & $-4^{\mathrm{m}} 43$ & 3.63 & 3.59 & (83) \\
\hline W599 & G9 & 3.10 & $0 \mathrm{~m}^{\mathrm{m}}$ & $1^{\mathrm{m}} 71$ & $8{ }^{m} 43$ & $0^{\mathrm{m}} 78$ & 3.733 & $-3^{\mathrm{m}} 66$ & $-4 \mathrm{~m}^{\mathrm{m}} 02$ & 3.46 & 3.39 & $90,(83)$ \\
\hline W601 & B1.5 & 3.50 & $0{ }^{\mathrm{m}} 60$ & $2^{\mathrm{m}} 10$ & $8^{\mathrm{m}} 56$ & $-0 \mathrm{~m}_{25}$ & 4.374 & $-3^{\mathrm{m}} 54$ & $-6{ }^{\mathrm{m}} 07$ & 4.28 & 4.27 & 93 \\
\hline $\mathrm{W} 605 \mathrm{sh}^{\star}$ & G2 & 3.10 & $0 \mathrm{~m}^{\mathrm{m}} 19$ & $0^{\mathrm{m}} 59$ & $11^{\mathrm{m}} 60$ & $00^{\mathrm{m}} 63$ & 3.768 & $-0 \mathrm{~m}_{50}$ & $-0 \mathrm{~m}^{\mathrm{m}} 70$ & 2.14 & 2.14 & (92) \\
\hline W606 & G9 & 3.10 & $0 \mathrm{~m}^{\mathrm{m}} 48$ & $1 \mathrm{~m}^{\mathrm{m}} 49$ & $9{ }^{\mathrm{m}} 03$ & $00^{\mathrm{m}} 78$ & 3.733 & $-3^{\mathrm{m}} 07$ & $-3^{\mathrm{m}} 43$ & 3.23 & 3.18 & (0) \\
\hline W611 & G8 & 3.10 & $0 \mathrm{~m}_{91}^{\mathrm{m}}$ & $2^{\mathrm{m}} \cdot 82$ & $9^{\mathrm{m}} 17$ & $00^{\mathrm{m}} 74$ & 3.746 & $-2^{\mathrm{m}} 93$ & $-3^{\mathrm{m}} 33$ & 3.19 & 3.09 & (13) \\
\hline W614 & B9 & 3.10 & $0^{\mathrm{m}} 71$ & $2{ }^{\mathrm{m}} 20$ & $10 \mathrm{~m}_{12}$ & $-0{ }^{\mathrm{m}} 07$ & 4.022 & $-1^{\mathrm{m}} 98$ & $-2^{\mathrm{m}} 49$ & 2.85 & 2.84 & (89) \\
\hline $\mathrm{W} 617^{\star}$ & K5 & 3.10 & $0^{\mathrm{m}} 41$ & $1 \mathrm{~m}^{\mathrm{m}}$ & $11^{\mathrm{m}} 02$ & $1^{\mathrm{m}} 15$ & 3.638 & $-1 \mathrm{~m}^{\mathrm{m}} 08$ & $-1 \mathrm{~m}_{80}^{\mathrm{m}}$ & 2.58 & 2.47 & (73) \\
\hline
\end{tabular}

From Fig. 4 it is evident that the distance modulus corresponding to a distance of $2.6 \mathrm{kpc}$ seems to be appropriate also for the PMS sample. We therefore conclude that this estimate is correct.

Before discussing the cluster membership of the sample of objects, we will first analyze one of the main criteria for this: the HRD. We start with the assumption that all the programme stars are located at the same cluster distance.

From the location of our 52 PMS candidates in the HRD, Fig. 4, we can separate three groups: Group I are all early-type stars, $(B-V)_{0}<\sim 0$ m 1 , and lie between the MS and one of the stellar birthlines. Group II contain the stars of intermediate spectral types, $\sim 0{ }^{\mathrm{m}} 3$ 


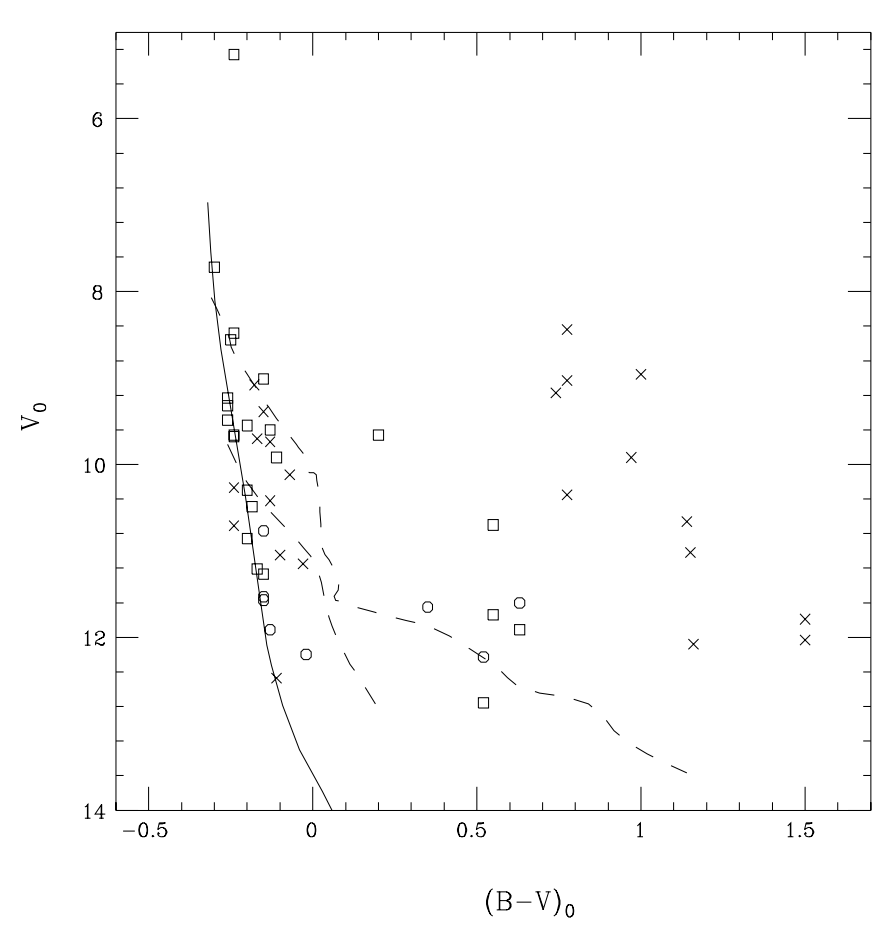

Fig. 4. The $V_{0},(B-V)_{0}$ diagram for our programme stars in NGC 6611. The data points plotted as $\times$ are of stars with $R_{V}=$ 3.1; those as $\square$ with $R_{V}>3.1$ and those as $\bigcirc$ are for stars with an IR-excess probably due to circumstellar dust radiation. The Groups I: $(B-V)_{0}<\sim 0$. 1 ; II: $\sim 0 .{ }^{\mathrm{m}} 3<(B-V)_{0}<\sim 0$. 7 ; and III: $(B-V)_{0}>\sim 0$. 7 are clearly separated

$<(B-V)_{0}<\sim 0$. 7 . They are located near or just to the right of the upper stellar birthline. Group III consists of the late-type stars with $(B-V)_{0}>\sim 0$ m 7 , which are located far to the right and/or far above the stellar birthline. We shall discuss now each group separately.

\section{Group I}

The early-type stars, the majority of our sample, fit nicely to the MS as derived by Thé et al. (1990) or to the ZAMS of Hillenbrand et al. (1993), or lie somewhat to the right of it. However, located to the right of the ZAMS we find the more luminous stars more frequently than the lower luminosity ones. This can be easily understood if we accept that several of these massive stars are already moving away from the ZAMS, to become post-MS stars.

The early-type stars at the lower end of the MS are remarkable. We find that 4 out of 5 of the early-type stars located in this part of the HRD have a strong near-IRexcess, probably due to dust. Although these objects are located near the ZAMS, they can still be young enough to belong to the HAeBe group. Furthermore, we see that for Group I stars there is no dependency of the $R_{V}$ value for their location in the HRD.

\section{Group II}

From this very interesting group none seem to have "normal" IR properties, either they have large $R_{V}$ values or they are probably surrounded by dust. Although these objects are located to the right of the birthline for large accretion rates one can not discard them as not being PMS if they are cluster members. As shown by Palla \& Stahler (1993) and in Fig. 4, it could be possible that if we change the accretion rate the location of a birthline could shift (it is discussed by Lamers et al. 1996, in preparation, that this can also happen by changing the metallicity of a star). For an accretion rate $\left(\dot{M}_{\text {acc }}>10^{-4} M_{\odot} \mathrm{yr}^{-1}\right)$ the Group $I I$ objects can be visible in their PMS phase.

\section{Group III}

All stars in this group have a normal extinction law, are located far to the right the birthline, are of very late spectral type and are relatively luminous if we place them at the cluster distance. The objects in this group are those objects for which Chini \& Wargau (1990) concluded that late-type stars are foreground objects. However, we have shown here (see Group $I I$ ) that we must discriminate between two types of late-type objects, depending on their IR properties and their location in the HRD.

The exceptional but interesting location of the star at $(B-V)_{0}=0{ }^{\mathrm{m}} 20$, is the star W213(1). The classification of this star used in option W213(2) is probably most appropriate one (see Table 7 and Sect. 7.2).

From Fig. 4 we encounter a major problem: where are the stars that approach the ZAMS?, or in other words: "where are the late A-type and F-type PMS objects?" We return to this interesting problem later.

In order to make this discussion more conclusive, we first discuss the probabilities of cluster membership for the programme stars. After which we discuss the extinction characteristics of the cluster field. Subsequently, conclusions are drawn in combination with the properties of the HRDs.

\section{Membership criteria}

Differentiating field stars from cluster members is crucial, but it is a quite complex problem. Tools to assist in separating them, are the $E(B-V)$ and probabilities of membership $(P)$ derived from proper motion studies (Thé et al. 1990). In this study, we add the location of an object in the HRD as a criterion of membership (see Fig. 5). This is because the assumed stellar evolutionary status for each star can be compared to the age of NGC 6611 of $5 \mathrm{Myr}$ or less. The resulting probability of membership, $P$ for most objects are listed in Table 7 . Note that such values are not always indicative to distinguish between cluster members and non-members. The proper motion of a star can be influenced by nearby cluster members, binarity, or by its formation process. 
In the direction of NGC 6611 a distance of $2.6 \mathrm{kpc}$ implies $E(B-V) \approx 0.50$ (Thé et al. 1990 and Fig. $2)$. A star being a cluster member must therefore have $E(B-V)>0.50$. Background objects in the cluster field will have a larger $E(B-V)$-value. From Fig. 2 and from Fig. 1 of Thé et al. (1990) we take an upper limit of $\sim 0.8$. Indeed most of our objects have an $E(B-V)$ value between 0.55 and 0.85 , see Table 7 . We refer to this group of stars as Group A.

A second group, Group $B$, with 0.9 $<E(B-V)<1^{\mathrm{m}} 0$, can be identified from the same figures. A third group, Group $C$, are objects which have very large $E(B-V)$ values, up to $1^{\mathrm{m}} 63$. Finally, we distinguish Group $D$ as objects with $E(B-V)<0$ m 50 , being probably non-cluster members.

In this section, we meticulously examine Groups $A-D$ and deduce their membership probabilities.

\subsection{Stars in Group A}

7.1.1. Stars with a high proper motion membership probability

From Table 7 it appears that a large number of stars having an $E(B-V)$ value between 0.55 and $0^{\mathrm{m}} \cdot 75$ also have high proper motion membership probability. From their position in the HRD, we find that most of these objects also belong to Group I. We will consider these MS stars as confirmed cluster members and are indicated "Y" in Table 8. However, two stars need a closer analysis:

W266sh: it has a good $E(B-V)$ value for membership, $0 \mathrm{~m} 68$, but no proper motion of this star is known. W266sh is located just above the stellar birthline (Group II) in the HRD. The shell designation is due to its IR-excess above the F8 SED. The origin of this excess remains doubtful. If it is due to CS dust then the object is a good protostar-candidate. However, the CS extinction will reduce the IS value close to a foreground value. In case of an M-companion we have no such problem. We adopt this object as a probable cluster member.

W599: is definitely not a cluster member, since it is a very bright star that lies far away to the right of the stellar birthline. It has a late spectral type (G9). It cannot be at the same time a cluster member and an evolved object. It has also a rather low $E(B-V)$ value, 0.555 , which suggest membership. However, its location in the HRD proves it to be a foreground object.

\subsubsection{Stars with a low proper motion membership probability}

In Group $A$ we have also a number of stars whose $E(B-V)$ value indicates that they are cluster members, but their proper motion probability suggests the opposite. The objects are: W240, W299, W300, W374, W396a, W396b,

\section{W440, W455 and W494sh.}

W240: $E(B-V)=0{ }^{\mathrm{m}} 67, P=0 \%$ and Sp.T. $=$ B8. It is located low in the HRD, but still on the MS. It seems reasonable to conclude that W240 is a probable cluster member.

W299sh: $E(B-V)=0{ }^{\mathrm{m}} 83, P=0 \%$ and Sp.T. $=\mathrm{B} 6$. We find W299sh located next to the ZAMS (Group $I$ ) which suggests that it is probably a cluster member.

W300: shows again a good membership value $E(B-V)$ $=0.77$. It has an early spectral type, B2. In the HRD W300 is located exactly on the MS. Although Kamp's (1974) $P$ value is $0 \%$, it is a possible cluster member.

W374: $E(B-V)=0^{\mathrm{m}} \cdot 56$ is a boundary case. We have derived F2 for its spectral type. Examining the HRD, we find W374 (option: $R_{V}=4.4$ ) to lie close to the stellar birthline at the right side (Group II) and even on the stellar birthline when we plot W374sh. Due to the possible presence of CS dust, the IS extinction can be lower than the total value of 0.56 . In case of anomalous extinction this value is a minimum (note that $E(B-V) \propto R_{V}^{-1}$ ). This indicates that we should be careful in accepting this star to be a cluster member, but if this is the case, it seems to be a very good PMS-candidate.

W396: we have to consider both options with $P=0 \%$ : W396a (Sp.T. $=$ F9, $E(B-V)=0$ m 64$)$ and W396b (Sp.T. $=\mathrm{G} 2, E(B-V)=0.56)$. We find both options located to the right of the stellar birthline (Group II) in the HRD. In both cases, the position in the HRD and the late spectral types suggest that the star is a very young object. The alternative would be that W396 is a foreground object. This seems to be unlikely if we consider the derived $E(B-V)$ values and the presence of anomalous extinction.

W440(1): with $E(B-V)=0 .{ }^{\mathrm{m}} 61, P=0 \%$ and spectral type G9, it is located in the HRD far away and above the stellar birthline. Because of this and its late spectral type it cannot be an evolved cluster members, see Fig. 9 . Together with the fact that we found a normal extinction law, $R_{V}=3.1$, we conclude that this star is not a cluster member as is the case for most of such objects, see Fig. 4.

W455: $E(B-V)=0.75, P=7 \%$ and Sp.T. $=$ B7. In the HRD W455 is located between the stellar birthline and the MS-curve (Group I), which makes it very possibly a cluster member, although the SED fit shows a depletion in the near-IR.

W494sh: has a $1 \%$ membership probability according to its proper motion, $E(B-V)=0.73$ and its spectral type is B6. Examining the star's location in the HRD, we find it is located just above the MS. We can likely assume 
that this star is a cluster member and most probably a good PMS-star candidate due to the presence of CS dust.

\subsection{Stars in Group B}

As far as their $E(B-V)$ is concerned, this group is located at the border of the "back" side of NGC 6611. However, if we consider that the extinction consist of an IS, CS and intracluster (IC) value, they could be very well cluster members. Group B consists of W213(2), W245sh, W297, W306, W406 and W611.

W213: we have two options with unknown probability of membership: W213(1), with $E(B-V)=1{ }^{\mathrm{m}} \cdot 27$ and spectral type A7 (this option belongs strictly speaking to Group $C$ but we also consider it here); and W213(2), with $E(B-V)=1$ m 01 and spectral type $\mathrm{F} 9$. Comparing the two obtained SED fits, we concluded that option W213(2) is better. In the HRD, W213(1) lies isolated, quite high and to the right of the stellar birthline. W213(2) is located somewhat lower than W213(1) in the HRD, but also to the right of the stellar birthline. W213(2) appears to belong to Group II. The HRD positions of W213(1) and W213(2) and the SED fits permit us to discard option W213(1) and only to consider W213(2) from now on. W213(2) is probably a very young object and probably a cluster member.

W245sh: shows evidence for CS dust, it has: $E(B-V)$ $=0.92, P=0 \%$ and spectral type B6. In the HRD we find W245sh reasonably close to the ZAMS (Group I). This star could be a suitable HAeBe candidate and considered to be a probable cluster member as we have no contradictory proof.

W297: $E(B-V)=0 \cdot 94, P=0 \%$ and spectral type B1. Based on its location in the HRD (Group I), the presence of an anomalous extinction law and the $E(B-V)$ of the star, it is highly probable that W297 is a cluster member.

W306: it has $E(B-V)=0.90, P=89 \%$ and spectral type B2. W306 belongs to Group I and we assume that it is a cluster member.

W406: $E(B-V)=0$ m 88 , spectral type K0 III and $P$ $=0 \%$. In the HRD W406 is seen as a very bright star that lies far to the right of the stellar birthline (Group III). We conclude, therefore, that W406 is not a cluster member and most probably a background object.

W611: $E(B-V)=0.91$, spectral type is G8 and $P=13 \%$, is located above and far away to the right of the stellar birthline in the HRD. W611 is therefore a Group III object and probably no cluster member. As for W406 the extinction law is normal, we therefore must conclude that it is a background object.

\subsection{Stars in Group $C$}

Stars in this group are expected to be background objects or objects that are located in a dark region (high IC extinction) or with a considerable amount of CS material in the line of sight (high CS extinction). Stars that exhibit an $E(B-V) \approx 1{ }^{\mathrm{m}} 1$ or more, are: W188, W202, W213(1), discussed above, W339(2), and W556.

W188: has the largest $E(B-V)$ value of our sample: 1. 63 . Its membership probability is $87 \%$. It has been classified as B0. In the HRD we find W188 next to the ZAMS, which makes it acceptable to presume that W188 is a cluster member. This is supported by the small anomalous extinction.

W202: $E(B-V)=1$. 13 , spectral type B3 and $P=$ $0 \%$. In the HRD we find W202 located next to the ZAMS. This implies that W202 is a probable cluster member.

W339(2): $E(B-V)=1$. $^{\mathrm{m}} 08$, spectral type B3 and proper motion not known. Furthermore we obtained two fits. In the HRD W339(2) $\left(R_{V}=3.9\right)$ is located between the ZAMS and the stellar birthline (Group I). The other fit (W339(2)sh), in which a dust shell was fitted, is located much closer to the ZAMS. Although this fit is not as good as that for W339(2) it must be considered because it makes a good HAeBe candidate. However, note that for W339(2) the IR-excess can be explained by an M-type companion also. For both options we conclude that this star is most probably a cluster member.

W556: $E(B-V)=1$ m $43, P=93 \%$ and spectral type B2. In the HRD we note that W556 is the brightest star of our sample and it lies somewhat above, although in the proximity of, the MS. W556 appears to be a cluster member, and is probably evolving away from the MS.

All of these three objects have a slightly anomalous extinction, indicating that they are located in or behind a region with larger average dust grains than that of the IS material. The objects seem to be cluster members but located in regions with high IC absorption.

\subsection{Stars in Group D}

Stars in this group are considered to be foreground objects as they have very low $E(B-V)$ values $(<\sim 0.50)$. Most of these objects, W103, W232, W349, W402, W411, W525, W534, W605, W606 and W617, belong to Group III, have a high membership probability according to their proper motion, but are located high and/or far to the right of the upper stellar birthline in the HRD. We therefore discard W349, W402, W411, W525, W534, W606 and W617 as cluster members. They are rated "N" in Col. 2 of Table 8. We discuss the objects W103, W232 and W605 as their 
location in the HRD does not permit us to draw a direct conclusion.

W103: $E(B-V)=0{ }^{\mathrm{m}} 43, P=90 \%$ and spectral type B9. Although there is an IR-depletion in the SED fit, W103 is located between the ZAMS and the stellar birthline in the HRD which would indicate it to be a cluster member with a luminosity class V. Perhaps the depression in the IR-photometry is due to absorption lines. Probably W103 is a foreground object. Otherwise, it could be a PMS star.

W232: $E(B-V)=0{ }^{\mathrm{m}} 39$, spectral type $\mathrm{F} 8$ and no known proper motion. It is located below the stellar birthline in the HRD. No definite conclusions can be made on whether this is a cluster member or not. Its $E(B-V)$ value clearly suggests that it is a foreground object, but the presence of anomalous extinction and traces of emission lines indicate it could be young. In case it is a cluster member it could be a PMS candidate of which the $E(B-V)$ is strongly affected by the anomalous extinction or the detected variability.

W605sh: $E(B-V)=0.19, P=92 \%$ and spectral type G2. In the HRD, we locate W605sh on the right side close to the stellar birthline. We conclude that this star is probably a foreground object surrounded by a dust shell, which would make it a very interesting object to study, or it could have a background IR-source.

The membership probabilities discussed in this section are listed in Table 8. In Fig. 5 we make a comparison of the membership probabilities of an object and its location in the HRD. From this figure it is evident that most of the Group III stars are foreground objects, as confirmed before. W599 has an $E(B-V)$-value close to the foreground one, and W611 has a low probability of membership, both probably being foreground stars. The Group I and Group II objects contain both populations. This makes it clear that it is best to discuss each star individually, as done above.

\section{The nature of the anomalous extinction in NGC 6611}

For each programme star the final SED fit yielded an individual extinction law correlated to one unique $R_{V}$ value as listed in Steenman \& Thé (1991). Although it could be well possible that other sets of extinction laws, when available, could produce other $R_{V}$ values (note that the extinction laws used are normalized to $E(B-V)$ ), however, the important conclusion will remain that the extinction law changes from star to star. This is in contradiction with an averaged value valid for the whole cluster or parts of it as discussed in the introduction and confirms a similar conclusion by Thé et al. (1990).

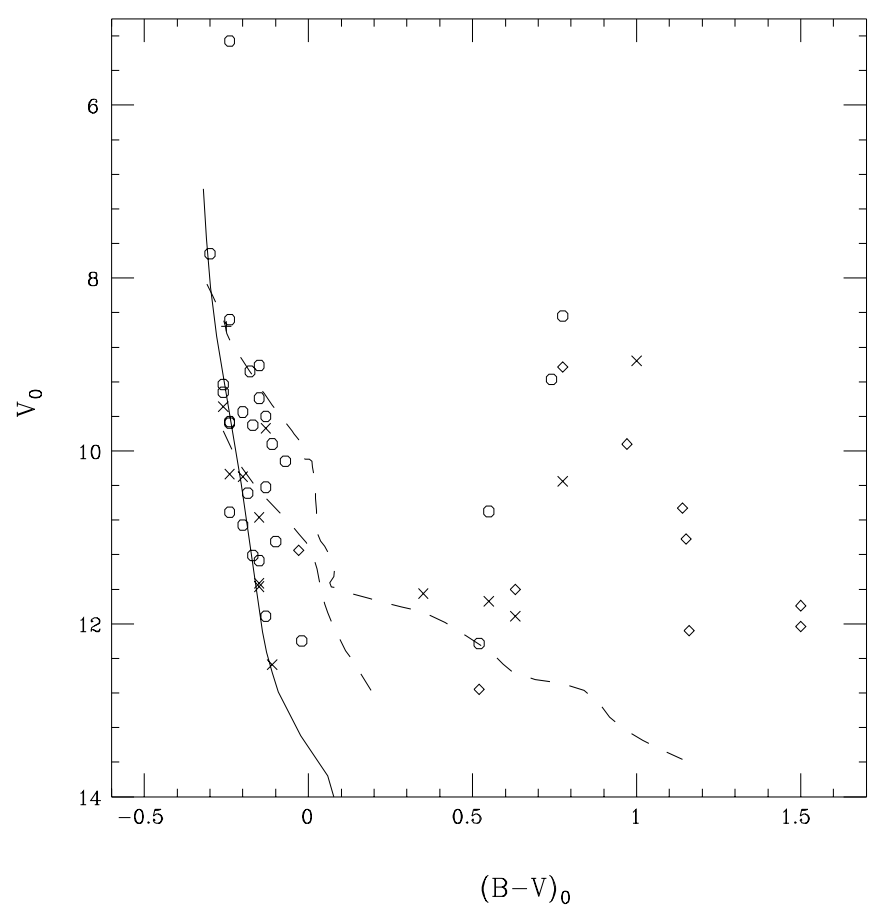

Fig. 5. The $V_{0},(B-V)_{0}$ diagram for our programme stars in NGC 6611 that have different probabilities of membership. The stars being probably nonmembers are indicated as a $\diamond$ in case $E(B-V)<0$. 50 , or as $\times$ when $P<10 \%$. The remaining cases, probably being cluster members, are noted as $O$

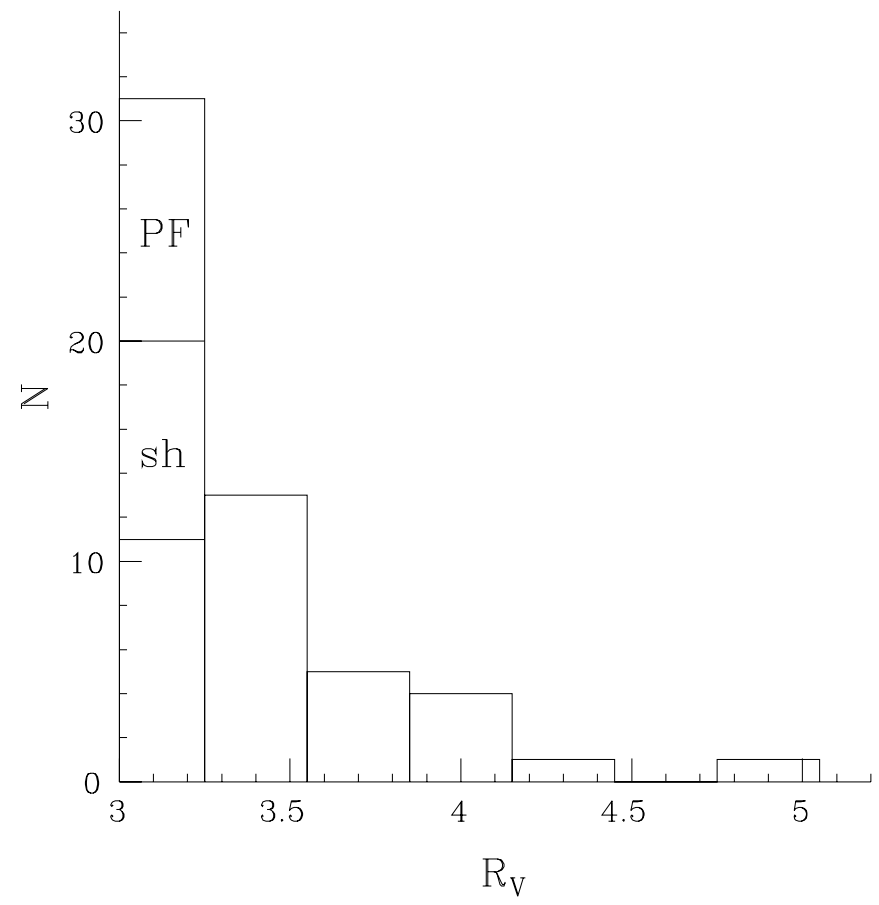

Fig. 6. Histogram of $R_{V}$ values. Part of the first column are the $s h$ cases, for which we adopted $R_{V}=3.1$, and possible foreground $(\mathrm{PF})$ objects 
Table 8. Membership probabilities, Col. 2, of the programme stars in NGC 6611 as discussed in the text. Typical Herbig $\mathrm{Ae} / \mathrm{Be}$ characteristics are also given. The abbreviations used are: $\mathrm{P}=$ probably; $\mathrm{Y}=$ yes; $\mathrm{N}=$ not; $\mathrm{d}=$ dust; $\mathrm{M}-\mathrm{C}=$ M-Companion

\begin{tabular}{|c|c|c|c|c|}
\hline Name & Membership & Variable & Emission & IR-Excess \\
\hline W103 & $\mathrm{PN}$ & & & \\
\hline W188 & PY & & & $\mathrm{M}-\mathrm{C}$ \\
\hline W202 & PY & & $\mathrm{e}$ & \\
\hline W207 & $\mathrm{Y}$ & & e: & \\
\hline W213(2) & PY & $\mathrm{Y}$ & e: & \\
\hline W232 & $?$ & $\mathrm{Y}$ & $\mathrm{e}$ & high $R_{V}$ \\
\hline W240 & PY & & & \\
\hline W245sh & PY & $\mathrm{Y}$ & $\mathrm{e}$ & d \\
\hline W262sh & PY & & & $\mathrm{M}-\mathrm{C}$ \\
\hline W266sh & PY & $\mathrm{Y}$ & e: & $\mathrm{d} / \mathrm{M}-\mathrm{C}$ \\
\hline W267 & $\mathrm{Y}$ & & $\mathrm{e}$ & \\
\hline W273sh & $\mathrm{Y}$ & $\mathrm{Y}$ & e: & $\mathrm{M}-\mathrm{C}$ \\
\hline W276(1) & $\mathrm{Y}$ & & & \\
\hline W290 & $\mathrm{Y}$ & & & \\
\hline W297 & PY & & $\mathrm{e}$ & \\
\hline W299(1) & PY & & e: & high $R_{V}$ \\
\hline W299(1)sh & PY & & e: & M-C \\
\hline W300 & PY & & e: & \\
\hline W306 & $\mathrm{Y}$ & & e & \\
\hline W313 & $\mathrm{Y}$ & $\mathrm{Y}$ & $\mathrm{e}$ & \\
\hline W336(2) & $\mathrm{Y}$ & & $\mathrm{e}$ & \\
\hline W339(2) & PY & & $\mathrm{e}$ & high $R_{V}$ \\
\hline W339(2)sh & PY & & $\mathrm{e}$ & $\mathrm{M}-\mathrm{C}$ \\
\hline W349 & $\mathrm{N}$ & & & \\
\hline W374sh & PY & $\mathrm{Y}$ & $\mathrm{e}$ & high $R_{V}$ \\
\hline W388 & $\mathrm{Y}$ & $\mathrm{Y}$ & e: & high $R_{V}$ \\
\hline W396(a) & PY & & e: & \\
\hline W396(b) & PY & & & \\
\hline W400 & $\mathrm{Y}$ & & $\mathrm{e}$ & high $R_{V}$ \\
\hline W402 & $\mathrm{N}$ & & & \\
\hline W406 & $\mathrm{N}$ & & & \\
\hline W411 & $\mathrm{N}$ & & & \\
\hline W440(1) & $\mathrm{PN}$ & & & \\
\hline W455 & PY & & $\mathrm{e}$ & \\
\hline W469 & $\mathrm{Y}$ & & & \\
\hline W483 & $\mathrm{Y}$ & & $\mathrm{e}$ & \\
\hline W484 & $\mathrm{Y}$ & & e: & \\
\hline W489 & $\mathrm{Y}$ & & & \\
\hline W494sh & PY & $\mathrm{Y}$ & $\mathrm{e}$ & d \\
\hline W500 & $\mathrm{Y}$ & $\mathrm{Y}$ & $\mathrm{e}$ & \\
\hline W504 & $\mathrm{Y}$ & & e: & \\
\hline W520 & $\mathrm{Y}$ & & & \\
\hline W525 & $\mathrm{N}$ & $\mathrm{Y}$ & & \\
\hline W534 & $\mathrm{N}$ & $\mathrm{Y}$ & e: & \\
\hline W536 & $\mathrm{Y}$ & & & \\
\hline W556 & PY & & & \\
\hline W559 & $\mathrm{Y}$ & & & \\
\hline W599 & $\mathrm{N}$ & & & \\
\hline W601 & $\mathrm{Y}$ & & & \\
\hline W605sh & $\mathrm{PN}$ & $\mathrm{Y}$ & & d \\
\hline W606 & $\mathrm{N}$ & & & \\
\hline W611 & $\mathrm{N}$ & & & \\
\hline W614 & Y & & & \\
\hline W617 & $\mathrm{N}$ & Y & e: & \\
\hline
\end{tabular}

As a next step it would be interesting to investigate whether the different extinction laws depend on the star's location in the cluster and to see whether they are connected to the spectral type.

To show the total "amount" of anomalous extinction, a histogram is drawn for the $R_{V}$ values from Table 7 . The first interval was chosen to be $3.0 \leq R_{V} \leq 3.2$, based on the normal extinction law with $R_{V}=3.1 \pm 0.1$ (see also Savage \& Mathis 1979). The following intervals all have a range of 0.3 (a value slightly larger than the error spread), with statistics up to $R_{V}=5.0$. The results are drawn in Fig. 6.

More than $50 \%$ of our objects have normal extinction. And besides two exceptions, the $R_{V}$ value is not extreme, in the sense $\gg 4.0$. Note that we have distinguished in the first interval of Fig. 6 the sh cases, for which we have adopted a normal extinction law, and the (probable) noncluster members from Table 8 . This reduces the total number of cluster objects with a normal extinction law from 32 to 11 . We also have to notice that some stars have been counted double, as we treated different options as being different objects. Because of the different extinction laws found for these cases, the statistics will not change much. In this respect, we mention that the two cases with extreme $R_{V}$ values (W273 and W374) are included here and not their sh options as discussed in Sect. 5 .

What remains besides the 11 objects with normal extinction, are 14 objects with a slightly different extinction law and 11 with very different extinction laws. No peak around some $R_{V}$ value seems to be present. This would indicate a more or less equal anomalous extinction, throughout NGC 6611.

To know the anomalous extinction distribution in NGC 6611, quantitative and qualitatively, we used Figs. 1-2 of Walker (1961) and Fig. 9 and Table 1 of Kamp (1974) of the NGC 6611 field. The latter is reproduced in Fig. 7 in which our programme stars are indicated. In Fig. 7 the $R_{V}$ value for each of our sample stars is also given together with those of Thé et al. (1990), the mainsequence cases.

- First we consider the objects which exhibit a normal extinction law. All of those that are indicated as (probably) noncluster members in Table 8 occupy the eastern side of the central area and the outskirts of the cluster. They are all of late spectral type (Group III). From the sh options 7 out of 9 are located close to the central part of the central area of the cluster. Note that the sh option can also include very anomalous extinction. Five cluster members are scattered throughout the central area of the cluster (W240, W267, W300, W339 and W455). Of the remaining 7 stars, 6 are scattered in the eastern outskirts of the cluster and W103 at the south-west out of the central area. All of these objects are B-type stars (Group I). - Most of the objects having a slightly anomalous extinction law, $R_{V}=3.3-3.5$, occupy the central area of the cluster. But W536 lies in the south-east while W556 and W559 are located in the very west region of the cluster. All of these stars are (probably) cluster members and are of spectral type B. Exceptions are W213, A7 or F9, and W396, F9 and G2.

- The objects having a significant anomalous extinction law, $R_{V}=3.6-4.1$, are also located in the central area of NGC 6611, they are all (probable) cluster members and are of spectral type B. Exceptions are the B-type star W290 that is located down south of NGC 6611 , but is a 

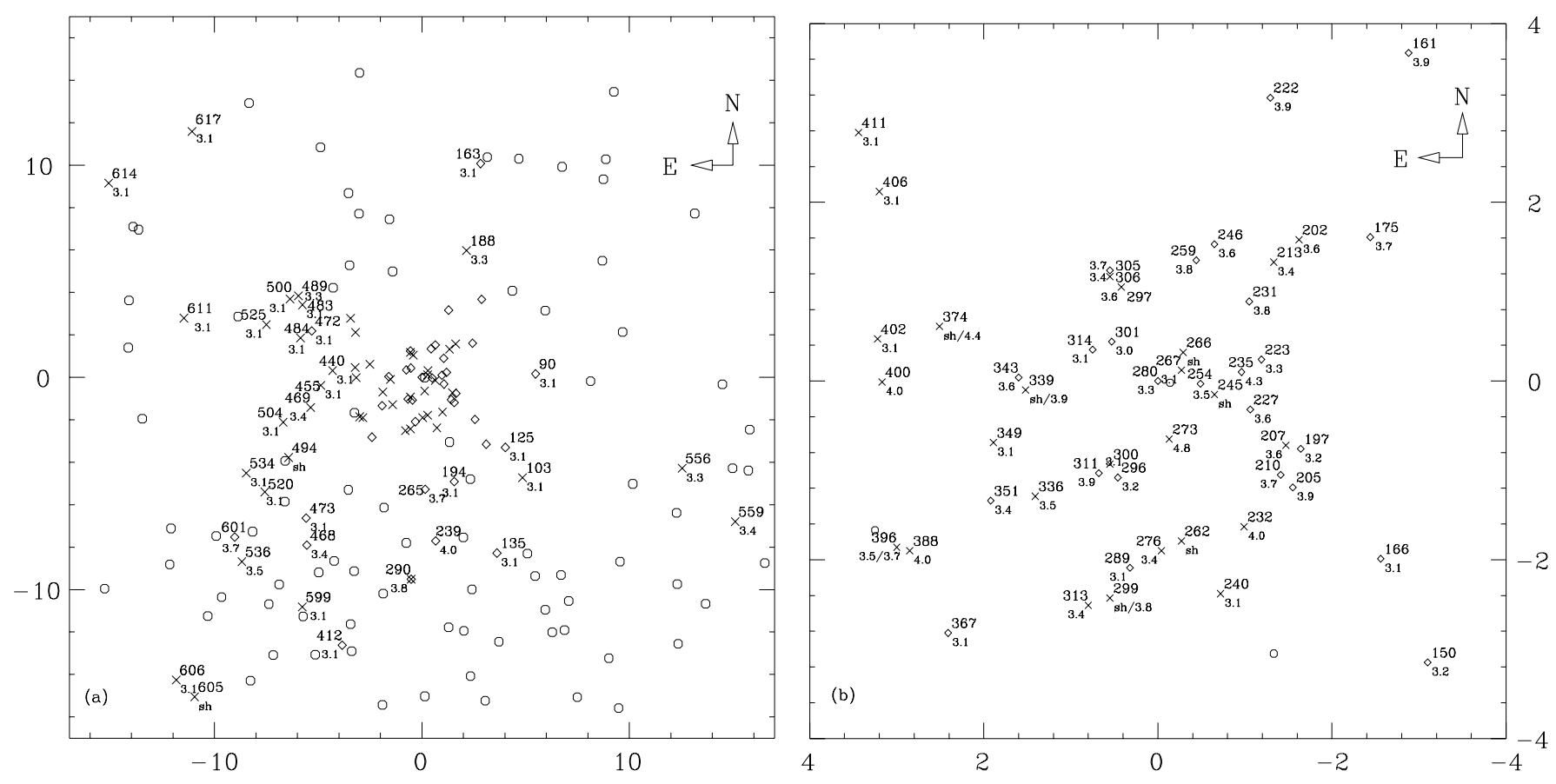

Fig. 7. Field of NGC 6111 reproduced from Fig. 9 \& Table 1 of Kamp (1974), a). The central $8^{\prime} \times 8^{\prime}$ area is enlarged in figure b). The PMS programme stars (indicated by crosses), as listed in Table 6, and the objects studied in Thé et al. (1990, diamonds) are indicated by their Walker number and by their individual $R_{V}$ value. Objects without a Walker number, the open dots, are other field stars. The scales are given in arcmin and centered on star W280 $\left(\alpha_{2000}=18^{\mathrm{h}} 18^{\mathrm{m}} 42^{\mathrm{s}} .84, \delta_{2000}=-13^{\circ} 46^{\prime} 51^{\prime \prime} .07\right)$

confirmed cluster member, W396 that is of spectral type F9 or G2 and W232 (F8), for which the membership is uncertain.

Besides a clear distribution of the later type stars with normal extinction laws to the East of the central area of NGC 6611, which are thought to be foreground stars, there seems no dependency of the extinction law or the place in the NGC 6611 areas. For example, as most of the sample stars are located in the central area, we do have neighbouring stars with normal and anomalous extinction. The variability in the $R_{V}$ values (from 3.3 to 4.0 , and possibly 4.4 or 4.8) suggests that the anomalous extinction law is very variable in the central area of the cluster. This is not surprising as we have already mentioned that the intracluster dust is unevenly distributed. Furthermore, hot stars (the B-types) can influence their direct circumstellar environment by evaporating and destroying larger grains, causing also large changes in the $R_{V}$ values of the intracluster material. The extinction law of a star located behind such a region will be altered as well. In cases of very young objects it is more likely that post-natal circumstellar material is the cause of a different than normal extinction. It is then interesting to note that Group II objects indeed does not have any members with a normal extinction law. This could imply that the anomalous extinction law is higher for late spectral types cluster members as they would occupy an earlier stage of their evolution than, for example, the averaged B-type members. The Group II objects could also be lying in a region where the intracluster matter is more dense, as they are all located in the central region.

Note also that most sh options, being of different spectral type, are located in the central part of the central area.

\section{Obscured and embedded sources in NGC 6611}

Knowing the result that each star has its own extinction law, which is therefore a local characteristic, these extinction laws will not provide additional information about the distribution of the dark material in NGC 6611. To obtain such information we have plotted values of $A_{V}$, the foreground value being up to $\sim 1^{\mathrm{m}} 5$, for each object as it is located in NGC 6611, Fig. 8. This figure contains also the MS programme stars from Thé et al. (1990). The $A_{V}$ values used are listed in Table 7 and calculated from Table 4 of Thé et al. (1990).

Although the combined sample is not high in number (88 objects), it is clear that the most obscured objects are located near the centre $(=$ W280 $)$ with a distribution of very high $A_{V}$ values to the north and to the northwest. Note that the field of NGC 6611, e.g. Fig. 7a, already shows that these regions are relatively less crowded. This would indicate that most dark material is distributed towards these directions and would be the region to look for young objects or even traces of star formation. 


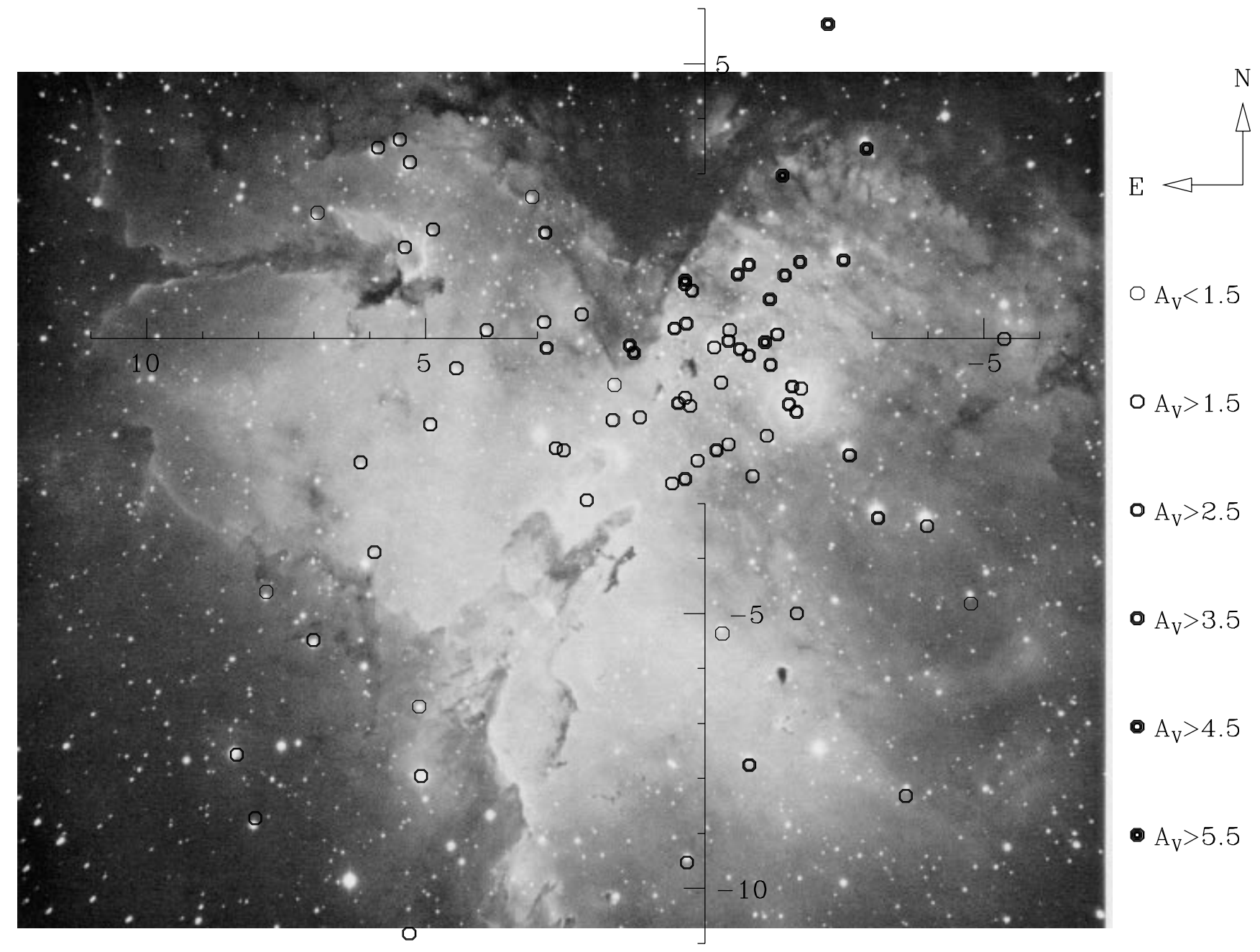

Fig. 8. Field of NGC 6111 reproduced from a Hale Observatory photograph of plates taken in $\mathrm{H} \alpha$ and $[\mathrm{N}$ II] $\lambda \lambda 6548,6583$ with the 200 inch Hale telescope (Osterbrock 1974). The bright H II region, M 16, is clearly visible. The central area of NGC 6611 is drawn by an overlay. We have indicated the $A_{V}$ values for the PMS-programme stars and the MS-objects of Thé et al. (1990) by an intensity scale, indicated at the right

Indeed, most embedded sources as discussed by Hillenbrand et al. (1993) are distributed towards the northeast of W280. This region would then be most important to look for on-going star formation. Hillenbrand et al. (1993) mention that more than $50 \%$ of the infrared objects located south or southeast are background objects. If we then refer to their Table 7 of embedded stars with circumstellar material, we find that many of these objects are located to the northwest of the stars with the highest $A_{V}$ values as plotted in Fig. 8. This supports our finding and indicates that most visible young objects seem to be distributed north-northwest of the centre (= W280) and on-going star formation could probably be occurring north-northeast of it.

\section{Emission-line objects in NGC 6611?}

The occurrence and variability of emission lines is one of the main characteristics of PMS objects, although it has been argued by Thé et al. (1993) that these lines will diminish in strength when arriving on the MS even though the objects are in their pre-ZAMS phase. Not all young objects will, therefore, directly show clear and strong emission lines. We have identified 29 objects with emission features in our sample, Table 4 , which is about $50 \%$ of the total sample or even $2 / 3$ rd if the non-cluster members are not taken into consideration. In the HRDs of Figs. 9a-b, the PMS candidates with emission or traces of emission features are indicated. Note the fact that most non-emission and emission line stars are not clearly distributed. Twenty seven emission line objects were also detected spectroscopically in the study of Hillenbrand et al. (1993), or about $35 \%$ of their sample of 79 objects.

As stated by Hillenbrand et al. (1993), it is a well known difficulty to calibrate emission lines for a star in a cluster such as NGC 6611. The bright "background" nebula will exhibit strong but sharp emission lines. If the star is located in or behind such a nebula it is very 
challenging to make an appropriatea subtraction of the nebular emission. A good subtraction is only possible on long-slit CCD exposures (Horne 1986) as obtained by us. Another difficulty could be the absorption in the cluster region. If a programme star is located in the cluster, part of the radiation in the hydrogen lines will be absorbed by dark inter-cluster material. It is, therefore, questionable whether the stellar emission is strong enough to be detected. Whether these conjectures are realistic seem to be suggested by the contradicting results of the IDS and CCD data. Eleven objects are detected to have emission lines in one but not in both of the spectra. Conclusions of the PMS nature of a star based on the absence of emission lines, and considering its line variability, should be treated carefully. Consequently, it is difficult to compare detections of emission line objects with those of other samples, such as the "MS-sample" as plotted in Fig. 9b. Additional spectra are necessary, especially of the more luminous objects, to detect emission features related to the evolutionary process.

The presence of emission lines does not seem to be related to the location in the cluster and the presence of anomalous extinction or an IR-excess, which confirms a similar finding by Hillenbrand et al. (1993).

Note that the spectrum of $W 339$ seems to be of symbiotic origin. Apart from lines indicating the existence of an early type object, some wide absorption bands seem to be present which could indicate the presence of a $\mathrm{T}$ Tauri companion. This companion could explain the presence of emission lines other than those of hydrogen.

\section{The evolutionary status of the objects in NGC 6611}

In previous studies the presence of PMS stars in NGC 6611 was disputed. Some authors (e.g. Walker 1961) believe that they can be identified from their location in the HRD. New insights, however, show that stars located in the Walker (1961) PMS-part of the HRD are probably never visible as PMS (Palla \& Stahler 1992 and 1993), as they are located far above the birthline. Others believe that the "Walker PMS" stars are all foreground objects, e.g. Chini \& Wargau (1990). Because of the youth of NGC 6611 (5 10 $10^{6}$ yr, Thé et al. 1990), one expects to detect at least some PMS objects. A comparison of this age with the evolutionary tracks of Palla \& Stahler (1993) for stars in their contraction phase and which move in the HRD towards the ZAMS, learns that the intersection of such tracks with the ZAMS for the age of NGC 6611 lies at stars with an initial mass of $2.5-3.0 M_{\odot}$. The intersection is at about $\log T_{\text {eff }}=4.1$, corresponding to spectral type B7 and is an upper limit as Hillenbrand et al. (1993) discussed the existence of one $6 \mathrm{Myr}$ old object in NGC 6611. However, most other objects in their study are suggested to be considerably younger, $2 \pm 1 \mathrm{Myr}$, indicating the possibility of an age spread in NGC 6611.
In Fig. 9a we have plotted our sample of PMS candidates in a theoretical HRD. The transformation of the $V_{0}$ versus $(B-V)_{0}$ diagram to the $\log L_{\star} / L_{\odot}$ versus $\log T_{\text {eff }}$ diagram is done as explained in Sect. 6 of Thé et al. (1990), adopting a distance modulus of $V_{0}-M_{V}=12$. 1 . Results of this transformation, such as $M_{V}$ and $\log L_{\star} / L_{\odot}$, are given in Table 7. Hillenbrand et al. (1993) argued that due to the quantization of the luminosity classes the bolometric corrections can yield a very significant error in the determination of the luminosities and finally in the masses and conclusions of the evolutionary status. To avoid this, we have determined the scaled stellar luminosity by integrating over the entire fitted Kurucz model (de Winter \& van den Ancker 1996). This method results in values of $L_{\star} / d^{2} L_{\odot}$ and is less sensitive to small errors in adopted spectral types and luminosity classes. These SED luminosities are also listed in Table 7 by adopting a distance of $2.6 \mathrm{kpc}$.

In Fig. 9a we have also plotted the theoretical results of Palla \& Stahler (1993). Note that in their calculations a star with $6 M_{\odot}$ already reaches the ZAMS within $0.1 \mathrm{Myr}$. Compared to the birthlines most objects could be well in their PMS-phase if we adopt a general accretion rate onto the very young objects of $\dot{M}_{\mathrm{acc}}=10^{-4} M_{\odot} \mathrm{yr}^{-1}$. In such a circumstance the objects with $M_{\star}>6 M_{\odot}$ and located right of the ZAMS must be extremely young and certainly not of the cluster age.

At a cluster age of a few million years PMS-objects must be of spectral type B7 or later. Stars of earlier spectral type have therefore ended their PMS phase and are normal main-sequence stars, assuming that there is no age-spread. Such objects will slowly move to the right on the main sequence band. An object of $40 M_{\odot}$ will have left this band already within 5 million years (Maeder \& Meynet 1988). This would mean that the spread of the early type objects right of the ZAMS in Fig. 9b is due to this post-ZAMS evolution. In this figure we have plotted our sample stars as well as those of Thé et al. (1990), as they are cluster members probably being in their MS phase, together with evolutionary tracks of Maeder \& Meynet (1988). As the MS-band is even filled up to stars with $60 M_{\odot}$ we must conclude that these stars are either still very young, less than 3 million years, or evolved as they have ended their red-giant phase which they do in about 10 Myr.

Thé et al. (1990) concluded that the estimated age was reliable compared to other estimates. A weak point involved in the age-determination is the assumption that all stars are at the same distance, furthermore, new calculations can be used to compare with our results. According to such recent theoretical calculations, the distribution in an HRD of the studied NGC 6611 members indicate that either the objects are of different ages or that either all the objects are considerably younger, about one Myr, as many objects are located close to the birthlines, see Fig. 9a. In that case stars with $M_{\star}>4 M_{\odot}$ will be either 

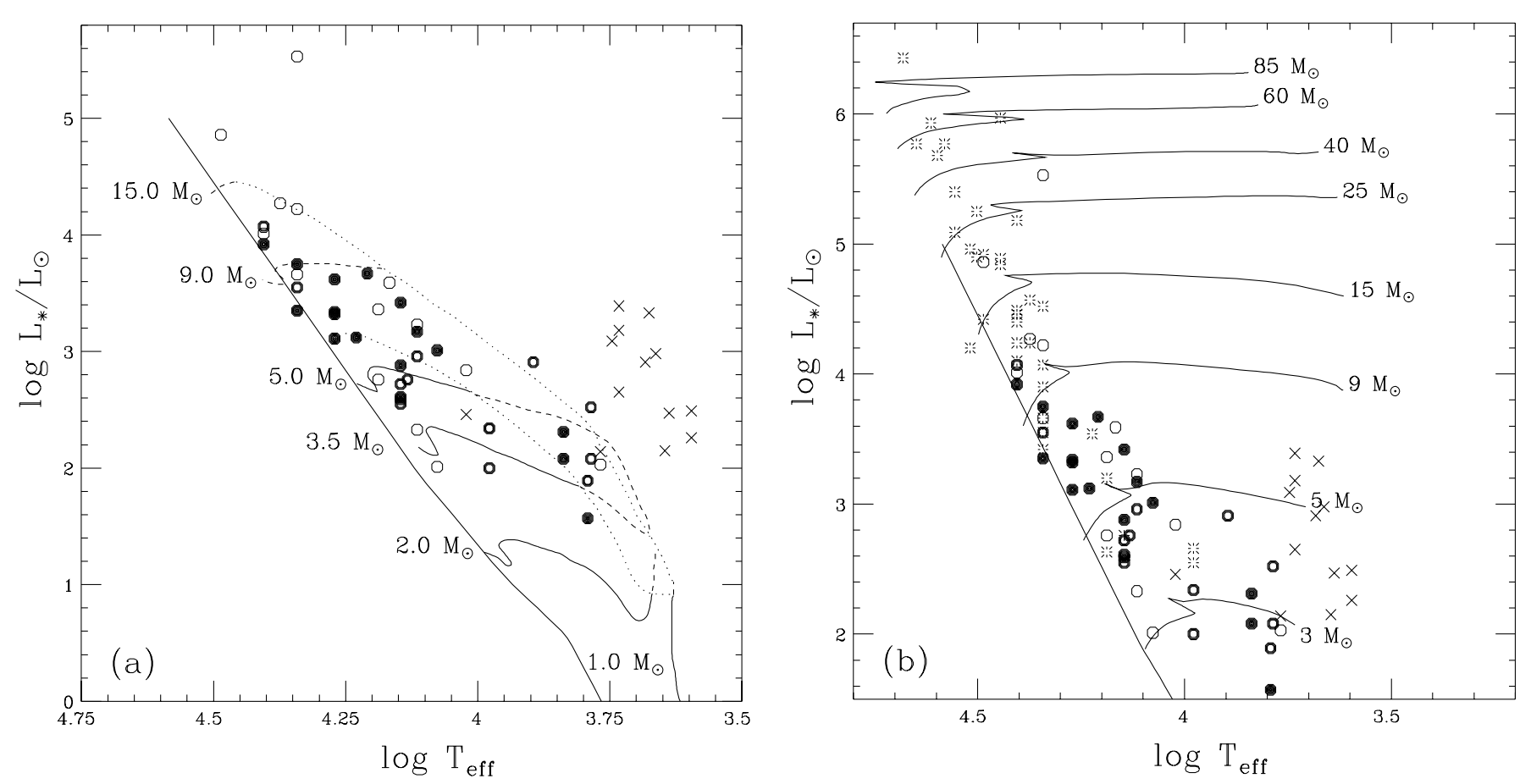

Fig. 9. HR-diagram with the positions of the sample of PMS-candidates of NGC 6611 as taken from Table 7. The emission-line stars are indicated by solid dots. Stars with possible emission by half open dots and the open dots the non emission-line objects. They are compared to: a) The pre-main sequence evolutionary tracks and birthlines, dotted, of Palla \& Stahler (1993); b) The post-main sequence evolutionary tracks of Maeder \& Meynet (1988) together with the results of Thé et al. (1990) on the MS-candidates of NGC 6611, indicated by stars. Crosses indicate the objects which are not or probably not cluster-members as listed in Table 8. The zero-age main-sequence from Hillenbrand et al. (1993) is also given. The latter does not suite the theoretical results in all the cases

extremely young or will be in their post-MS phase. In the latter case such objects, that are located close to the right border of the MS in Fig. 9b, or close to the stellar birthlines in Fig. 9a, are then of ages close to about 100 million years (Maeder \& Meynet 1988), leaving enough time for the most massive objects to have ended their "life". The only way out of this paradox is to assume an age spread in NGC 6611, as already proposed by Hillenbrand et al. (1993).

The three exceptional cases, with $\log L_{\star} / L_{\odot}>5.4$, which are located to the very right of the MS, see Fig. 9b, are then evolved massive objects with ages of $4-6 \mathrm{Myr}$. These ages also confirm the location of objects with $5 M_{\odot}$ and the ones close to the ZAMS; they are post-ZAMS. The objects located far to the right of the ZAMS are then in their pre-ZAMS phase having ages of a few $0.1 \mathrm{Myr}$. This would explain the situation of the Group II objects as being all just formed with $M_{\star}<4 M_{\odot}$.

As the distribution of the studied objects in the field of NGC 6611 in Figs. 9a-b do not show any clear turn-off point, this confirms an age spread. This is more dramatically seen in Fig. 9 of Hillenbrand et al. (1993). Only if we include the Group II objects, we have a type of turn-off point but still with some points between these objects and the ZAMS. It would be these intermediate objects that are slightly older, a few million years, than the Group II objects, and, than the more massive objects located right of the ZAMS.

The age spread of less than one million year up to about 6 million years also indicates that for finding preZAMS or pre-MS objects it is best to look at characteristics of individual cases as in earlier findings of our sample.

\section{Discussion and conclusions}

To arrive at our goal of detecting PMS stars, we made use of many observations, of which the numerous photometric and spectroscopic datasets are the most important. With the different photometric datasets we were able to sort out the poor quality observations and measurement errors as well as to select observations taken at the same brightness level. This provided us with reliable data for investigating the SED of each programme star. Combined with the fact that we determined the spectral types of the cluster members using different TCDs combined with spectra, we were able to discuss and determine accurate stellar astrophysical parameters. The photometric data was 
dereddened with the use of these parameters and was fitted to an appropriate theoretical Kurucz (1991) model.

A first result of this fitting procedure was the detection of an individual extinction law for each star. We analyzed the extinction laws and concluded that they are not correlated with the stellar spectral type, nor they depend on the location of the star in the cluster field. Indeed, stars located near the central area, do show more often a higher $R_{V}$ value, i.e. anomalous extinction. However, the normal and anomalous extinction can sometimes be found in other regions of the cluster as well, and varies from one star to a neighbouring one. The anomalous extinction can, therefore, be explained as being a very local effect or patchy in nature. Possibly, the stellar radiation influences the circumstellar or nearby intracluster material. The finding of individual extinction laws rather than an averaged one for the whole cluster, is in strong contradiction with previous studies. In such studies, the extinction law was based on TCDs. However, as shown by Steenman \& Thé (1989), an extinction law is not defined by three colours, but it must be determined by using colours from the blue to the near-IR. The approach which makes use of the TCDs to determine extinction laws and or $R_{V}$ values is therefore only indicative. Our approach affects the determination of the stellar $A_{V}$ and $V_{0}$ and hence alters the stellar location in the HRD and conclusions derived from it.

The SEDs are also used to identify peculiarities in the energy distribution of each star. Likewise, we identified weak and strong IR-excesses and even depletions in the dereddened near-IR. The near-IR depletions could be fitted to Kurucz models by taking higher luminosity classes for late type objects, but could also be due to strong absorption bands or observational errors. The weak nearIR excess could be well fitted with anomalous extinction. A strong near-IR excess was found for nine stars. Possible explanations are the detection of circumstellar dust or nearby or background M-type stars, located in the aperture used for the photometry. However, free-free emission, due to the gaseous inner parts of circumstellar material, could not be excluded as the cause of IR-excess.

One of the main PMS characteristics is the occurrence of a near-IR-excess due to thermal dust emission of (a) circumstellar shell(s). Analyzing the temperatures of each near-IR-excess, by comparing them to black-bodies, we concluded that W245, W266, W494 and W605 are the only ones that could have circumstellar dust. The other five strong near-IR emitters have either an M-type companion, need very high $R_{V}$ values (very anomalous extinction) or could have a large amount of free-free emission. Checking the programme stars for other PMS characteristics, we noted 29 objects with possible stellar emission lines in their spectra. Of the stars that possibly have a shell around them are W245, W339, W374 and W494 emission line stars. W266, W273 and W299, three other stars with a strong near-IR excess, probably have emission lines. Another PMS characteristic, the variability of the stellar brightness, could not be analyzed due to an insufficient number of data points for most stars. However, considering the ranges for the stars having a strong nearIR-excess, besides W339 and W494, all have $\Delta V>0 \mathrm{~m} 2$. Because W339 and W494 are B-type stars, this result is not in contradiction with the results of Bibo \& The (1991) regarding the variability of PMS stars.

To verify whether we have detected PMS objects, young stars, we have to place them in an HRD. After computation of $V_{0}$, using the individual $R_{V}$ value, and $(B-V)_{0}$ for each star, we composed an HRD in which we could identify three stellar groups from their location in this diagram, see Fig. 5. Group I, the early-type stars, are located on or near the MS. Group II consists of stars having a later spectral type, up to late G, whereas Group III consist of stars having even a later spectral type or a higher luminosity.

Group III stars are all identified to be foreground objects, due to their low $E(B-V)$, their low probability of membership and their location in the HRD, which cannot be explained by stellar evolution considering the probable youth of the cluster. This confirms only part of the conclusion of Chini \& Wargau (1990) that the Walker (1961) PMS candidates are all foreground objects.

More interesting are the Group I and Group II objects. Group I, the early-type stars, coincides with the Hillenbrand et al. (1993) sample which has a cut-off at A0. They are all located near the transformed ZAMS from Hillenbrand et al. (1993), using and confirming the cluster distance of $2.6 \pm 0.2 \mathrm{kpc}$, as derived in Thé et al. (1990). Furthermore, we do not find any stars located to the very right of the MS-band in our pre-MS sample, Fig. 9a. But if we include the MS-sample of Thé et al. (1990), Fig. 9b shows three objects located right of the MS having an age of about $4-6 \mathrm{Myr}$ in case they are evolving towards the red-giant phase. Adopting an age of $5 \mathrm{Myr}$ of the cluster (Thé et al. 1990), we must have located a clear turn-off point at about $25 M_{\odot}$ in Fig. 9b or Fig. 8 of Hillenbrand et al. (1993). The early-type stars located at the right of the ZAMS, close to the upper stellar birthline, must therefore be younger. If they are still in their pre-MS phase, it is remarkable that they do not show typical HAeBe characteristics.

HAeBe candidates, however, are found among the lower luminosity early-type stars, four have a very strong near-IR emission. Two of these stars have IR-excess temperatures possibly indicative of dust (W245 and W494), the other two, W262 and W273, have excess temperatures of roughly $2500 \mathrm{~K}$, which is an upper limit. We conclude from these findings that the emission line stars W245 and W494 are indeed HAeBe stars. As we noticed, they have the PMS characteristics as mentioned before. W262, without any emission lines, and W273, do show some PMS properties, but must be studied further for their IR-properties. W262 has an IR-excess due to stellar dust and it would be interesting to verify whether the 
spectrum is indeed without any emissions as can be disputed from Fig. 1. The PMS candidates we found are of spectral type B6 or later. Compared to the location right of the ZAMS and the evolutionary tracks of Palla \& Stahler (1993) these objects cannot be older than 1 Myr.

The situation is more complex for the Group II objects. Some of these stars do fulfill all membership criteria, whereas others do not, see Fig. 5. All have a peculiar nearIR characteristic, W605 and W266 have CS dust emission, the other ones have anomalous extinction laws. Two objects of Group II have a very low $E(B-V)$, the other four have not. The location of the Group II objects, very close to the stellar birthline with the highest accretion rate, can be explained by the hypothesis that these stars have just been formed. Compared to the calculations of Palla \& Stahler (1993), pre-main sequence objects of 3.5 to $5.0 M_{\odot}$ younger than a few $0.1 \mathrm{Myr}$ can occupy this region of the HR-diagram. Normally, such stars are hardly visible due to their very high $A_{V}$ as they are very much embedded in their post-natal cloud. For the stars of Group II we could not find such values of $A_{V}$, except for W213 $\left(A_{V}=4 .{ }^{\mathrm{m}} 3\right)$. Due to the fast rate of their formation, there must be an efficient mechanism clearing the post-natal and shell or disk material. If a shell or disk is formed, as proposed by Hillenbrand et al. (1993), this must happen within 1 Myr. Such an effective clearing of the circumstellar environment of the young star supports the hypothesis that at least part of the Group I objects are also in their pre-main sequence phase without showing typical HAeBe characteristics, normally due to circumstellar material.

The mentioned objects of Group I and Group II do fulfill the stellar birthline of Palla \& Stahler (1993) for protostars with an accretion rate of $\dot{M}_{\text {acc }}=10^{-4} M_{\odot}$ $\mathrm{yr}^{-1}$. As these objects must have ages of about a few times $0.1 \mathrm{Myr}$ and do not show evidence of any circumstellar material, it could be that the disk survival time is of an order of $10^{4}-10^{5}$ yrs, if a disk is formed from a shell left over at birth, or it seems that the NGC 6611 stars are built up at a faster rate than generally assumed for most known HAeBes.

Objects of different ages are found in NGC 6611, indicating that the sample objects are not coeval. Hence, we conclude that star formation has an age spread. This was also indicated by Hillenbrand et al. (1993), who discovered many embedded objects, probably in formation as they are still optically thick. It would be of high interest to study whether the natal material is enriched and what are its effects on the early stellar evolution compared to the young evolved objects. The effective clearing of the CS environment could then be due to stellar evolution on a shorter time scale due to the presence of an enhanced metallicity.

Note that the "gap" between Group I and Group II objects can be explained by the differences in age of the cluster population. As the lower-MS objects are about a few Myr, the ones of Group II are significantly younger.
In fact we have an age-gap. However, we have to note the few objects in this part of the HRD as we suffer from an observational cut-off at lower magnitudes and a cut-off in the Hillenbrand et al. (1993) paper at A0. An additional study of objects of A- and F-type stars being in their PMS-phase must be considered as a main follow-up study. Studying these objects, filling the "gap", could give more insight in the theory and understanding of stellar formation and especially the way they become optically visible. Note in this respect the discussion in Hillenbrand et al. (1993) that many emission-line objects in NGC 6611 look like classical $\mathrm{Ae} / \mathrm{Be}$ type stars rather then having typical HAeBe characteristics. This adds to the confusion of the definitions for these different groups of emission-line objects. The efficient clearing of the dust of the young objects in NGC 6611 could explain why we observe young classical-like Ae/Be type stars.

A possible way to clean the CS environment of young stars could be by the radiation of the already evolved massive early type members of the cluster. Especially as the central part of NGC 6611 is crowded with such objects, the UV radiation field will be very destructive. In this respect the discovery of evaporating gaseous globules (EGGs) by Hester \& Scowen (1995) could also well explain our findings as such bodies probably do not contain enough angular momentum to distribute the post-natal material from a shell to a disk-like structure. The impact of star formation by EGGs in this cluster on our results will be discussed in more detail in a forthcoming paper.

Although we have reached our ultimate goal by finding at least three PMS Be-candidates, probably typical HAeBe stars, our main conclusion is that we just started to understand the stellar formation and early evolutionary phases and they appeared to be different and more complex than generally assumed.

\section{References}

Bibo E.A., Thé P.S., 1991, A\&AS 89, 319

Brand J., Wouterloot J.G.A., 1988, A\&AS 75, 117

Chini R., Krügel E., 1983, A\&A 117, 289

Chini R., Wargau W.F., 1990, A\&A 227, 213

Grinin V.P., Thé P.S., de Winter D., et al., 1994, A\&A 292, 165

Hester J., Scowen P., 1995, NASA Press Release No. 95-190

Hillenbrand L.A., Massey P., Strom S.E., Merrill K.M., 1993, AJ 106, 1906

Horne K., 1986, PASP 98, 609

Jacoby G.H., Hunter D.A., Christian C.A., 1984, ApJS 56, 257 Johnson H.L., Borgman J., 1963, Bull. Astron. Neth. 17, 115 Johnson H.L., 1966, ARA\&A 4, 193

Kamp L.W., 1974, A\&AS 16, 1

Koulis C., 1993, Student Dissertation, Univ. of Amsterdam

Kurucz R.L., 1991, Precision photometry; Astrophysics of the Galaxy, A.G. Davis Philip, A.R. Upgren, K.A Janes (eds.). L. Davis press, Schenectady, New York

Lub J., Pel J.W., 1977, A\&A 54, 137

Maeder A., Meynet G., 1988, A\&AS 76, 411 
Neckel Th., Chini R., 1981, A\&AS 45, 451

Osterbrock D.E., 1974, Astrophysics of Gaseous Nebulae, Freeman and Company, San Fransisco

Palla F., Stahler S.W., 1990, ApJ 360, L47

Palla F., Stahler S.W., 1992, ApJ 392, 667

Palla F., Stahler S.W., 1993, ApJ 418, 414

Pel J.W., 1987, Internal Rep. Leiden Obs

Sagar R., Joshi U.C., 1979, Ap\&SS 66, 3

Savage B.D., Mathis J.S., 1979, ARA\&A 17, 73

Schmidt-Kaler Th., 1982, Landolt Börnstein Catalogue, Vol. $\mathrm{VI} / 2 \mathrm{~b}$

Steenman H., Thé P.S., 1989, Ap\&SS 159, 189
Steenman H., Thé P.S., 1991, Ap\&SS 184, 9

Thé P.S., Cuypers H., Tjin A Djie H.R.E., Feinstein A., Westerlund B.E., 1985, A\&A 150, 345

Thé P.S., de Winter D., Feinstein A., Westerlund B.E., 1990, A\&AS 82, 319

Thé P.S., de Winter D., Pérez M.R., 1993, A\&AS 104, 315

Turner D.G., 1974, PhD Thesis, Univ. of Western Ontario, Canada

Von Schewick H., 1962, Veröffentl. Sternwarte Bonn, No. 62

Walker M.F., 1961, ApJ 133, 438

de Winter D., Thé P.S., 1991, MmSAI 62, 881

de Winter D., van den Ancker M.E., 1996, A\&A (in press) 